\title{
A Modified Nonlinear Modal Synthesis Scheme for Mistuned Blisks with Synchronized Switch Damping
}

\author{
Jiuzhou Liu $\mathbb{D}^{1,2,3}$ Lin Li, ${ }^{1,2,3}$ Yu Fan $\mathbb{D}^{1,2,3}$ and Xingrong Huang ${ }^{4}$ \\ ${ }^{1}$ School of Energy and Power Engineering, Beihang University, 37 Xueyuan Road, Beijing 100191, China \\ ${ }^{2}$ Collaborative Innovation Center for Advanced Aero-Engine, 37 Xueyuan Road, Beijing 100191, China \\ ${ }^{3}$ Beijing Key Laboratory of Aero-Engine Structure and Strength, 37 Xueyuan Road, Beijing 100191, China \\ ${ }^{4}$ Centrale Pekin, Beihang University, 37 Xueyuan Road, Beijing 100191, China
}

Correspondence should be addressed to Yu Fan; fanyu04@buaa.edu.cn

Received 3 March 2018; Accepted 8 May 2018; Published 12 July 2018

Academic Editor: Giovanni Palmerini

Copyright (c) 2018 Jiuzhou Liu et al. This is an open access article distributed under the Creative Commons Attribution License, which permits unrestricted use, distribution, and reproduction in any medium, provided the original work is properly cited.

In the authors' previous work (MSSP 2017), we show that the synchronized switch damping based on negative capacitor (SSDNC) is a good candidate for vibration suppression of tuned blisks. In this paper, we consider the mistuned case and propose an efficient numerical approach to accelerate the required statistical analysis. Although SSDNC is a type of nonlinear piezoelectric damping, through an in-depth nonlinear modal analysis, we show that the modal information of the system remains unchanged with respect to the nonlinear modal amplitude. Based on this, an accelerated nonlinear component modal synthesis (NCMS) method is proposed to predict and further analyse the dynamic characteristics of the nonlinear system. The precision and efficiency of the proposed method is compared with that of the multiharmonic balance method. The stochastic characteristics of the blisk are studied with two sources of mistuning. The first one is random stiffness mistuning and the second one is capacitance mistuning. The investigation is carried out with different mistuning levels and under different engine-order excitations. The results show that the NCMS method can accurately predict the forced response of the mistuned blisk with SSDNC, and the calculation cost can be considerably reduced. Two advantages of the SSDNC technique applied to the mistuned blisk have been revealed in the statistical view. The first one is that the SSDNC can suppress the amplified vibration induced by the mistuning of the blisk significantly. The second one is that the vibration-suppression performance of SSDNC is insensitive to the mistuning of the blisk and that of the electrical circuits.

\section{Introduction}

Bladed disks are among key components in an aeroengine. They belong to a class of structures called periodic structures consisting of spatially repetitive substructures that are identical in the ideal situation [1]. However, in reality, there are always small and random deviations in blade properties, owing to factors such as manufacturing, material tolerances, and in-operation wear. These blade-to-blade discrepancies are called mistuning, which is typically small, but can lead to concentrated vibration in a small region of the structure. This phenomenon is known as vibration localization. As a result, certain blades in the mistuned system can have larger forced response levels than the ideal tuned design and will lead to an increase in vibration stress and premature high cycle fatigue (HCF) of the blades. HCF is a major cost, safety, and reliability issue for an aeroengine [2]. Therefore, it is important to suppress the amplification of the response induced by mistuning. The dry-friction damping has been the most common approach toward vibration mitigation since a long time [3-7]. However, this method is not favourable owing to the absence of contact interfaces in the integral bladed disk ("blisk" for short in the following text) in modern aeroengines. These issues drive researchers to find alternative damping techniques, particularly for the vibration suppression of a mistuned blisk with low structural damping.

In recent decades, piezoelectric materials have received extensive attention because of their properties such as light weight, high bandwidths, efficient energy conversion, and easy integration [8]. When embedded in or bonded to 
fundamental structures, piezoelectric materials can convert mechanical energy into electric energy and vice versa. On the basis of this energy conversion capability, a vibration reduction technique is developed [9]. Reference [10] investigated the possibility of dissipating mechanical energy with piezoelectric shunt circuits. They showed that the RL piezoelectric shunt circuit performs like a dynamic vibration absorber; therefore, the resistor and inductor can be optimally tuned to structural resonance in a manner analogous to a mechanical vibration absorber. References $[11,12]$ presented a vibration control method involving cyclic periodic structures using piezoelectric networks. It is shown that this approach can suppress all spatial harmonic components of the vibration, which cannot be achieved by the RL piezoelectric shunt circuit. Reference [13] considered the nonengineorder excitation from a practical point of view and explained the mechanisms for vibration suppression of a piezoelectric network and piezoelectric shunt circuit using modal analysis and energy analysis. References $[14,15]$ used a negative capacitor to improve the performance of piezoelectric networks. In addition, experiments were carried out to verify theoretical results. Reference [16] used the shape of the targeted mode to organize the piezoelectric patches as a modal filter, which decreases the necessary inductors in the shunted circuit. Their method was first illustrated experimentally on a circular plate and then used in a prototype of an industrial bladed drum. Reference [17] proposed a nonlinear piezoshunt circuit as a vibration absorber for the bladed disk. Both the nonlinear normal modes and nonlinear forced response of the electromechanical system were investigated theoretically. Their results show that when the bladed disk becomes mistuned, the nonlinear vibration absorber yields better vibration mitigation performance than the linear shunt circuit.

Except for the passive piezoelectric damping mentioned above, various semiactive piezoelectric damping techniques are being widely researched in recent years. Among them, the synchronized switch damping (SSD) technique has received considerable attention since it was proposed by Richard et al. [18, 19] because of its simple implementation and broadband performance. The piezoelectric patches, each one connected with an SSD circuit, are embedded in or bonded to the host structures, and the voltage difference between the electrodes is switched at the strain extrema or displacement extrema of the host structures in every vibration cycle. As the inversion of voltage is quite fast, the power consumed by the semiactive control system is usually low [20]. Based on the research on SSD, the synchronized switch damping on inductor $[21,22]$, the synchronized switch damping on voltage source $[23,24]$, and the synchronized switch damping on negative capacitor (SSDNC) [25] have been developed in the past ten years. Many switching control algorithms have been developed to maximize the energy dissipated in each cycle of vibration to improve the vibration suppression performance of the SSD methods [26-28]. Recently, self-powered SSD techniques have been proposed to make the entire system self-powered, and different types of control principles of the self-powered SSD are investigated to obtain better vibration suppression performance in a wideband frequency range [29-31]. Different from the SSD technique, Kauffman and Lesieutre [32] and Lopp and Kauffman [33] developed a concept of resonance frequency detuning (RFD) to simplify piezoelectric-based vibration reduction approaches and applied it on turbomachinery environment. As the excitation frequency of a certain engine order sweeps through a resonance crossing, a switch is made between stiffness states to detune the response from the excitation, resulting in a decrease in vibration.

These studies show that the SSD technique can suppress the vibration of multiple modes of structures, and there is no need for precise tuning of the electrical parameters. These characteristics are important to solve the vibration problems of the blisks in modern aeroengines, which generally have low structural damping and are excited over a wide frequency range. However, most of the existing studies on SSD are based on simple structures such as piezoelectric beams or plates, mainly used for experimental purposes. Research on the dynamic characteristics of the more complex systems such as the blisk with SSD is rare, and few results pertaining to the forced response of this type of system can be found because of the nonlinearity of the SSD. Considering the complexity and cost of experimental research on the blisk in aeroengines, it is meaningful and necessary to find an efficient method to predict the dynamic behaviours of the blisk with SSD. For these reasons, we investigated the dynamic characteristics of the blisk with the SSDNC using the multiharmonic balance method combined with the procedure of alternating frequency/time domain (MHBMAFT) [34]. With this method, we performed complex modal analysis and calculated the forced response of the nonlinear electromechanical system. The method was verified to have high calculation efficiency. However, these studies are aimed at the deterministic system (tuned blisk or blisk with a deterministic mistuning). In reality, the mistuning of the blisk is usually random and unpredictable. Therefore, a statistical analysis is necessary for the mistuned blisks to ensure suppression vibration. For this type of analysis, the main challenge is the high computing cost (memory consumption and computation time). Although the MHBM-AFT method used in our previous study can reduce the computation time significantly, it is still a challenge to carry out a Monte Carlo simulation with hundreds of random mistuning samples.

In this study, a nonlinear modal analysis of the blisk with the SSDNC reveals that the modal frequencies and modal shapes do not vary with the modal amplitude. Based on these properties, a nonlinear component modal synthesis (NCMS) method without subsequent iteration is proposed to predict the forced response of the blisk with the SSDNC, and its precision and efficiency are compared with the MHBM-AFT method. Then, the NCMS method is used to perform a statistical analysis on the mistuned blisk with the SSDNC to study the vibration suppression performance of the SSDNC from a statistical perspective.

The remaining parts of the paper are organized as follows: first, the lumped parameter model of the blisk and mathematical expression for the nonlinear force induced by SSDNC are presented (Section 2). Second, the dynamic equations of the nonlinear substructure are derived, a brief 


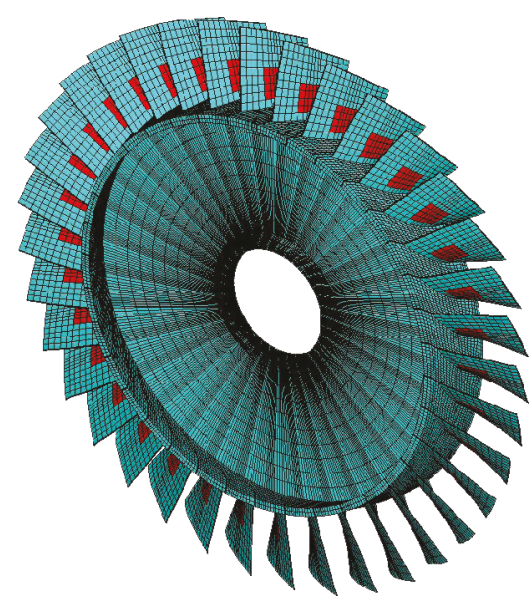

FIGURE 1: Illustration of the blisk with piezo-patches (the red region represents the piezo-patches).

description of the procedure for calculating the modal information of the nonlinear system is given, and the modal properties of the nonlinear substructure are discussed (Section 3). Then, the procedure for calculating the forced response of the nonlinear system in the frequency domain using the NCMS method (without subsequent iteration) is presented and its results are compared with the results obtained using MHBM-AFT (Section 4). Furthermore, a statistical analysis on the vibration-suppression performance of SSDNC and its sensitivity to mistuning of the blisk is presented (Section 5 ). Finally, the important findings of the study are summarized in the conclusion section (Section 6).

\section{Analysis Model and Nonlinear Force}

2.1. Lumped Parameter Model of Blisk. Figure 1 gives an illustration of the bladed disk with piezo-patches set on the surface of each blade root. When each piezo-patch is connected with an SSDNC circuit, such a blisk is simulated using a lumped parameter model in our study, as shown in Figure 2.

The blisk consists of $N$ sectors, and each sector is simulated by two blade-degrees-of-freedom (blade-DOFs) corresponding to two blade modes, and one disk-degreeof-freedom (disk-DOF) simulating one disk mode. The parameters $m_{\mathrm{b} 1}$ and $m_{\mathrm{b} 2}$ represent the mass of blade-DOFs, and $m_{\mathrm{d}}$ represents the mass of the disk-DOF. $k_{\mathrm{b} 1}$ and $k_{\mathrm{b} 2}$ represent the stiffness of the blade-DOFs, $k_{\mathrm{d}}$ represents the stiffness of the disk-DOF, and $k_{\mathrm{c}}$ denotes the coupling stiffness between adjacent sectors. $x_{\mathrm{b} 1}, x_{\mathrm{b} 2}$, and $x_{\mathrm{d}}$ denote the displacements of the two blade-DOFs and that of the diskDOF, respectively.

The piezo-element on the blade root (consisting of the piezo-patch and SSDNC circuit) is parallel to the spring $k_{\mathrm{b} 2}$ in the lumped parameter model. It should be noted that although in Figure 2, the mass and stiffness of the piezo-patch do not appear explicitly, they influence the dynamic behaviour of the blisk. Therefore, in the analysis, they are integrated with the mass $\left(m_{\mathrm{b} 2}\right)$ and stiffness $\left(k_{\mathrm{b} 2}\right)$ of the disk and represented by $m_{\mathrm{e}}$ and $k_{\mathrm{e}}$, respectively. They depend mainly on the usage amount and geometrical size of the piezo-patch.

2.2. Nonlinear Force Induced by SSDNC. As shown in Figure 2, the SSDNC circuit consists of a switch $S$, resistor $R$, and negative capacitor $C_{\mathrm{n}}$, all of which are connected in series. There are two states of the circuit in one vibration period of the structure: the closed state and the open state. The switch closes for a very short period at the maximum displacement of the fundamental structure, corresponding to the maximum voltage on the piezo-patch, and then opens. During the short closure time, the voltage is inverted because of the negative capacitor. Owing to the inverse piezoelectric effect of the piezoelectric materials, a force applied to the fundamental structure will be induced by the voltage on piezo-patch, and the force is proportional to the voltage.

Generally, the closed time of SSDNC is much smaller than the vibration period of the elastic structure and it is ignored in this paper. The force induced by the SSDNC is then expressed as follows [34]:

$$
\begin{aligned}
f_{\mathrm{nl}}\left(x_{\mathrm{b} 2, j}, x_{\mathrm{d}, j}, \dot{x}_{\mathrm{b} 2, j}, \dot{x}_{\mathrm{d}, j}, t\right)= & -\frac{1}{1-\chi_{\mathrm{c}}} \cdot \frac{\alpha^{2} x_{\mathrm{V}, j}^{\mathrm{M}}}{C_{\mathrm{p}}} \\
& \cdot \operatorname{sign}\left(\dot{x}_{\mathrm{b} 2, j}(t)-\dot{x}_{\mathrm{d}, j}(t)\right) \\
& +\frac{\alpha^{2}}{C_{\mathrm{p}}}\left(x_{\mathrm{b} 2, j}(t)-x_{\mathrm{d}, j}(t)\right),
\end{aligned}
$$

where $\alpha$ is the force factor and it can be obtained experimentally. When the materials of the piezo-patch are chosen, $\alpha$ depends on the usage amount, geometrical dimensions, and distributed localization of the piezo-patch. By optimizing these influence factors, a desirable value of $\alpha$ can be obtained. $\chi_{\mathrm{c}}=C_{\mathrm{p}} / C_{\mathrm{n}}$ is the capacitance ratio, where $C_{\mathrm{p}}$ is the inherent capacitance of the piezo-patch and $C_{\mathrm{n}}$ is the absolute value of the negative capacitor in the circuit. It can be seen from (1) that the force $f_{\mathrm{nl}}$ is a nonlinear function of the structural displacement and it is composed of two parts. Both the two parts are related to the displacement of the fundamental structure (the relative displacement of the blade root and disk in sector $j$ in Figure 2) and proportional to the same factor $\alpha^{2} / C_{p}$. However, the first part is proportional to $x_{\mathrm{V}, j}^{\mathrm{M}}$ (the amplitude of the relative displacement $\left(x_{\mathrm{b} 2, j}(t)(t)-x_{\mathrm{d}, j}(t)\right)$ and is multiplied by a factor $1 /\left(1-\chi_{\mathrm{c}}\right)$; therefore, by taking $\chi_{\mathrm{c}}$ to be approximately 1 , the direction of the nonlinear force is determined by its first part and is always opposite to the velocity of the fundamental structure.

The nonlinear force in the time domain can be seen in Figure 3(a), and the corresponding hysteresis loop (whose area represents the energy dissipated by the SSDNC in one vibration period) is illustrated in Figure 3(b).

\section{Modal Analysis of the Nonlinear Substructure}

3.1. Dynamic Equations for the Substructure of the Blisk with SSDNC. The blisk with the SSDNC can be sectioned into $N$ nonlinear substructures, as shown in Figure 4, and each substructure can be regarded as a nonlinear superelement. There 


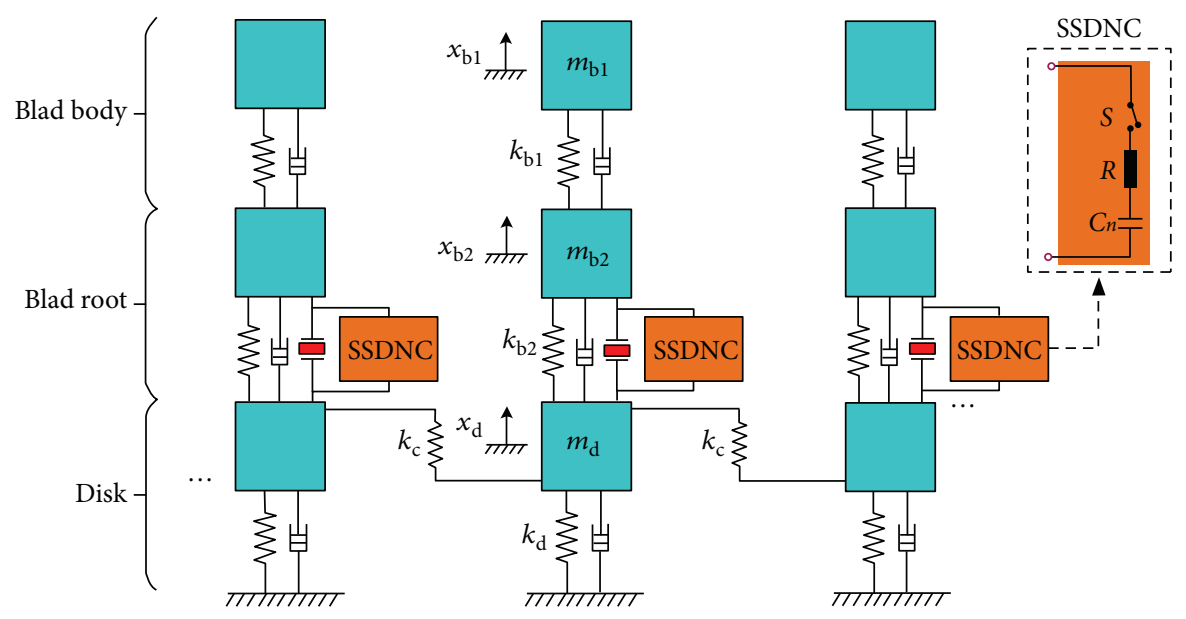

FIGURE 2: Lumped parameter model of the blisk with SSDNC.

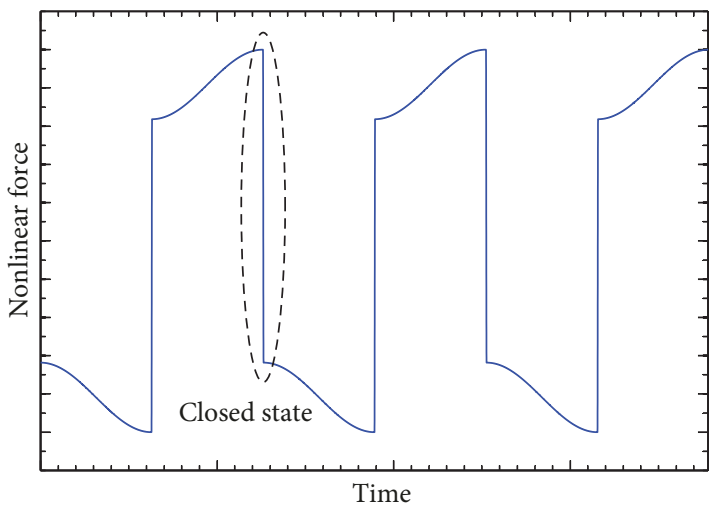

(a) Illustration of the nonlinear force in the time domain

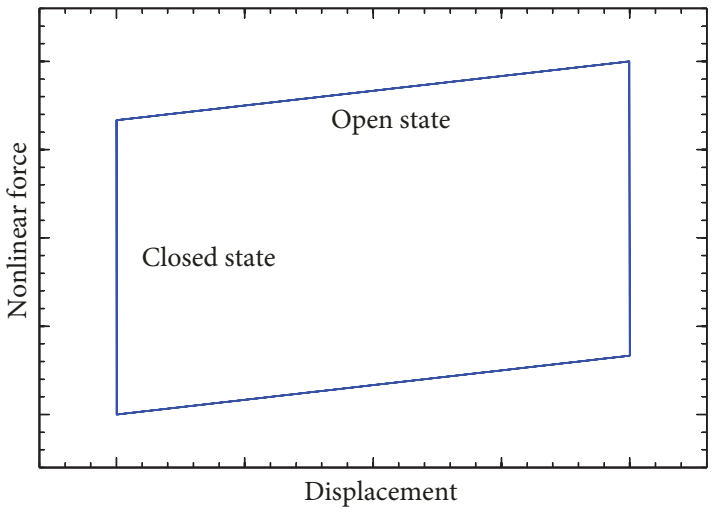

(b) Illustration of the hysteresis loop

Figure 3: Nonlinear force in the time domain.

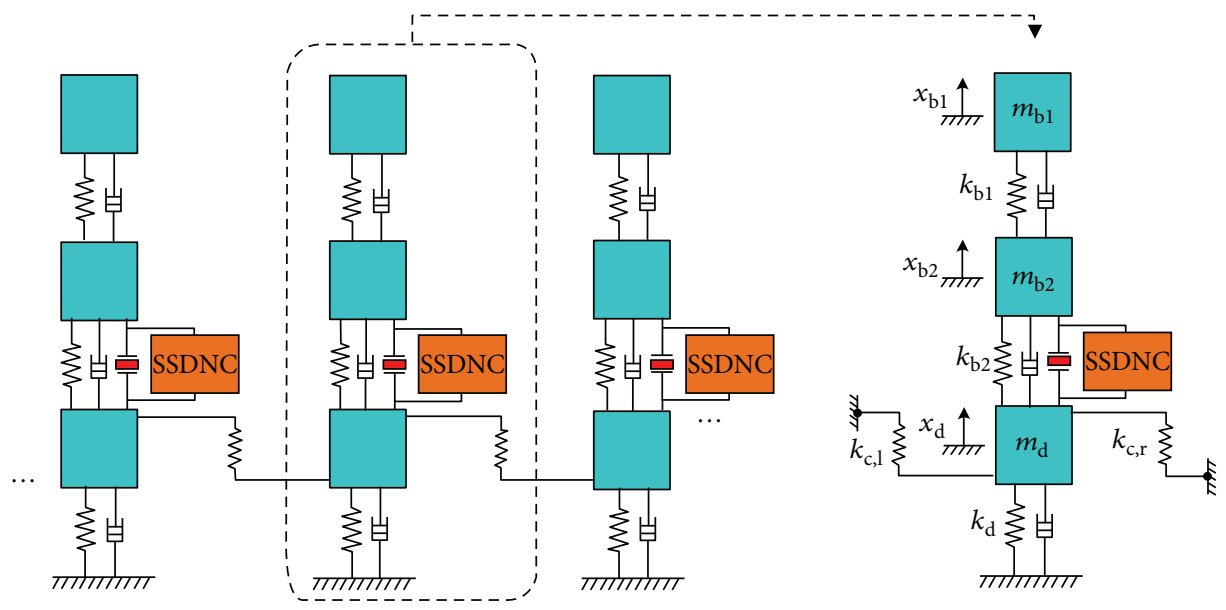

Figure 4: Nonlinear substructure.

are three internal DOFs and two boundary DOFs in one substructure. It should be noted that the mass of the two boundary DOFs $\left(m_{\mathrm{d}, \mathrm{l}}\right.$ and $\left.m_{\mathrm{d}, \mathrm{r}}\right)$ is zero. Because the periodic nonlinear blisk system can be obtained by connecting $N$ identical substructures in series, the stiffness (represented by $k_{c, 1}$ and $k_{c, r}$, resp.,) between $m_{\mathrm{d}}$ and the boundary DOFs equals to $2 k_{\mathrm{c}}$.

When the boundary DOFs are free, the nondimensional dynamic equations of the substructure without mechanical damping terms have the following forms: 


$$
\begin{aligned}
& \lambda^{2} \ddot{y}_{\mathrm{b} 1}+y_{\mathrm{b} 1}-y_{\mathrm{b} 2}=g(\tau), \\
& \lambda^{2}\left(\delta_{\mathrm{mb}}+\delta_{\mathrm{me}}\right) \ddot{y}_{\mathrm{b} 2}-y_{\mathrm{b} 1}+\left(1+\gamma_{\mathrm{b}}+\gamma_{\mathrm{e}}\right) y_{\mathrm{b} 2}-\gamma_{\mathrm{b}} y_{\mathrm{d}} \\
& +g_{\mathrm{nl}}\left(y_{\mathrm{b} 2}, y_{\mathrm{d}}, \dot{y}_{\mathrm{b} 2}, \dot{y}_{\mathrm{d}}, \tau\right)=\delta_{\mathrm{fb}} \cdot g(\tau) \text {, } \\
& \lambda^{2} \delta_{\mathrm{md}} \ddot{y}_{\mathrm{d}}-\gamma_{\mathrm{b}} y_{\mathrm{b} 2}+\left(\gamma_{\mathrm{b}}+\gamma_{\mathrm{e}}+\gamma_{\mathrm{d}}+4 \gamma_{\mathrm{c}}\right) y_{\mathrm{d}} \\
& -2 \gamma_{\mathrm{c}} y_{\mathrm{d}, \mathrm{r}}-2 \gamma_{\mathrm{c}} y_{\mathrm{d}, \mathrm{l}}=\delta_{\mathrm{fd}} \cdot g(\tau), \\
& \lambda^{2} \delta_{\mathrm{md}, \mathrm{r}} \ddot{y}_{\mathrm{d}, \mathrm{r}}-2 \gamma_{\mathrm{c}} y_{\mathrm{d}}+2 \gamma_{\mathrm{c}} y_{\mathrm{d}, \mathrm{r}}=0 \text {, } \\
& \lambda^{2} \delta_{\mathrm{md}, 1} \ddot{y}_{\mathrm{d}, \mathrm{l}}-2 \gamma_{\mathrm{c}} y_{\mathrm{d}}+2 \gamma_{\mathrm{c}} y_{\mathrm{d}, \mathrm{l}}=0 \text {. }
\end{aligned}
$$

The nondimensional nonlinear force induced by the SSDNC can be expressed as follows:

$$
\begin{aligned}
g_{\mathrm{nl}}\left(y_{\mathrm{b} 2}, y_{\mathrm{d}}, \dot{y}_{\mathrm{b} 2}, \dot{y}_{\mathrm{d}}, \tau\right)= & -\frac{1}{1-\chi_{\mathrm{c}}} \cdot \gamma_{\mathrm{p}} y_{\mathrm{V}}^{\mathrm{M}} \\
& \cdot \operatorname{sign}\left(\dot{y}_{\mathrm{b} 2}(\tau)-\dot{y}_{\mathrm{d}}(\tau)\right) \\
& +\gamma_{\mathrm{p}} \cdot\left(y_{\mathrm{b} 2}(\tau)-y_{\mathrm{d}}(\tau)\right),
\end{aligned}
$$

where $y_{V}^{\mathrm{M}}$ represents the maximum amplitude of the relative displacement between $y_{\mathrm{b} 2}$ and $y_{\mathrm{d}}$, and the dot above a letter represents the differentiation with respect to nondimensional time $\tau$. All the nondimensional parameters used above are defined as follows:

$$
\begin{aligned}
\tau & =\omega t, \\
\omega_{\mathrm{b} 1} & =\sqrt{\frac{k_{\mathrm{b} 1}}{m_{\mathrm{b} 1}},} \\
\lambda & =\frac{\omega}{\omega_{\mathrm{b} 1}}, \\
\gamma_{\mathrm{b}} & =\frac{k_{\mathrm{b} 2}}{k_{\mathrm{b} 1}}, \\
\gamma_{\mathrm{d}} & =\frac{k_{\mathrm{d}}}{k_{\mathrm{b} 1}}, \\
\gamma_{\mathrm{c}} & =\frac{k_{\mathrm{c}}}{k_{\mathrm{b} 1}}, \\
\gamma_{\mathrm{e}} & =\frac{k_{\mathrm{e}}}{k_{\mathrm{b} 1}}, \\
\gamma_{\mathrm{p}} & =\frac{\alpha^{2}}{\left(k_{\mathrm{b} 1} C_{\mathrm{p}}\right)}, \\
\delta_{\mathrm{mb}} & =\frac{m_{\mathrm{b} 2}}{m_{\mathrm{b} 1}}, \\
\delta_{\mathrm{md}} & =\frac{m_{\mathrm{d}}}{m_{\mathrm{b} 1}}, \\
\delta_{\mathrm{me}} & =\frac{m_{\mathrm{d}}}{m_{\mathrm{b} 1}}, \\
f_{\mathrm{b} 1}(\tau) & =f_{\mathrm{b} 1} \cdot g(\tau), \\
f_{\mathrm{b} 2}(\tau) & =f_{\mathrm{b} 2} \cdot g(\tau), \\
f_{\mathrm{d}}(\tau) & =f_{\mathrm{d}} \cdot g(\tau), \\
\delta_{\mathrm{fb}} & =\frac{f_{\mathrm{b} 2}}{f_{\mathrm{b} 1}},
\end{aligned}
$$

$$
\begin{aligned}
\delta_{\mathrm{fd}} & =\frac{f_{\mathrm{d}}}{f_{\mathrm{b} 1}}, \\
\bar{x} & =\frac{f_{\mathrm{b} 1}}{k_{\mathrm{b} 1}}, \\
y_{\mathrm{b} 1} & =\frac{x_{\mathrm{b} 1}}{\bar{x}}, \\
y_{\mathrm{b} 2} & =\frac{x_{\mathrm{b} 2}}{\bar{x}}, \\
y_{\mathrm{d}} & =\frac{x_{\mathrm{d}}}{\bar{x}} .
\end{aligned}
$$

Hence, the dynamic equations of the substructure can be written in the form of matrices:

$$
\lambda^{2} \mathbf{M} \ddot{\mathbf{y}}(\tau)+\lambda \mathbf{C} \dot{\mathbf{y}}(\tau)+\mathbf{K y}(\tau)+\mathbf{g}_{\mathrm{nl}}(\mathbf{y}(\tau), \dot{\mathbf{y}}(\tau), \tau)=\mathbf{g}(\tau) .
$$

The mass matrix $\mathbf{M}$ has the following form:

$$
\mathbf{M}=\left[\begin{array}{ccccc}
1 & 0 & 0 & 0 & 0 \\
0 & \left(\delta_{\mathrm{mb}}+\delta_{\mathrm{mp}}\right) & 0 & 0 & 0 \\
0 & 0 & \delta_{\mathrm{md}} & 0 & 0 \\
0 & 0 & 0 & \delta_{\mathrm{md}, \mathrm{r}} & 0 \\
0 & 0 & 0 & 0 & \delta_{\mathrm{md}, \mathrm{l}}
\end{array}\right] .
$$

The mass matrix $\mathbf{K}$ has the following form:

$$
\mathbf{K}=\left[\begin{array}{ccccc}
1 & -1 & 0 & 0 & 0 \\
-1 & \left(1+\gamma_{\mathrm{b}}\right) & -\left(\gamma_{\mathrm{b}}+\gamma_{\mathrm{e}}\right) & 0 & 0 \\
0 & -\left(\gamma_{\mathrm{b}}+\gamma_{\mathrm{e}}\right) & \left(\gamma_{\mathrm{b}}+\gamma_{\mathrm{e}}+\gamma_{\mathrm{d}}+4 \gamma_{\mathrm{c}}\right) & -2 \gamma_{\mathrm{c}} & -2 \gamma_{\mathrm{c}} \\
0 & 0 & -2 \gamma_{\mathrm{c}} & 2 \gamma_{\mathrm{c}} & 0 \\
0 & 0 & -2 \gamma_{\mathrm{c}} & 0 & 2 \gamma_{\mathrm{c}}
\end{array}\right] .
$$

When calculating the forced response, we introduce linear mechanical damping into the equations and the damping matrix $\mathbf{C}$ has the following form:

$$
\begin{aligned}
\mathbf{C} & =\alpha_{\mathrm{c}}\left[\begin{array}{ccccc}
1 & 0 & 0 & 0 & 0 \\
0 & \left(\delta_{\mathrm{mb}}+\delta_{\mathrm{mp}}\right) & 0 & 0 & 0 \\
0 & 0 & \delta_{\mathrm{md}} & 0 & 0 \\
0 & 0 & 0 & 0 & 0 \\
0 & 0 & 0 & 0 & 0
\end{array}\right] \\
& +\beta_{\mathrm{c}}\left[\begin{array}{ccccc}
1 & -1 & 0 & 0 & 0 \\
-1 & \left(1+\gamma_{\mathrm{b}}\right) & -\left(\gamma_{\mathrm{b}}+\gamma_{\mathrm{e}}\right) & 0 & 0 \\
0 & -\left(\gamma_{\mathrm{b}}+\gamma_{\mathrm{e}}\right) & \left(\gamma_{\mathrm{b}}+\gamma_{\mathrm{e}}+\gamma_{\mathrm{d}}\right) & 0 & 0 \\
0 & 0 & 0 & 0 & 0 \\
0 & 0 & 0 & 0 & 0
\end{array}\right],
\end{aligned}
$$


TABLE 1: Nondimensional parameters of the blisk.

\begin{tabular}{lcccccccccccc}
\hline Variable & $\delta_{\mathrm{mb}}$ & $\delta_{\mathrm{md}}$ & $\delta_{\mathrm{me}}$ & $\gamma_{\mathrm{b}}$ & $\gamma_{\mathrm{d}}$ & $\gamma_{\mathrm{c}}$ & $\gamma_{\mathrm{e}}$ & $\gamma_{\mathrm{p}}$ & $\alpha_{\mathrm{c}}$ & $\beta_{\mathrm{c}}$ & $\delta_{\mathrm{fb}}$ & $\delta_{\mathrm{fd}}$ \\
\hline Value & 1 & 12 & 0.05 & 1 & 1.5 & 30 & 0.05 & 0.01 & $10^{-6}$ & 0.05 & 1 & 0 \\
\hline
\end{tabular}

where $\alpha_{c}$ and $\beta_{c}$ are two constants characterizing the mechanical damping level.

3.2. Nonlinear Complex Modal Analysis Procedure. The nonlinear mode of motion is defined as an oscillation of the autonomous system with a phase difference between its degrees of freedom. Introducing the nonlinear mode provides a practical approach for the vibration analysis of nonlinear mechanical systems $[35,36]$. The modal information can be obtained using MHBM-AFT. The equation of an arbitrary nonlinear system without external excitation is as follows:

$$
\lambda^{2} \mathbf{M} \ddot{\mathbf{y}}(\tau)+\lambda \mathbf{C} \dot{\mathbf{y}}(\tau)+\mathbf{K y}(\tau)+\mathbf{g}_{\mathrm{nl}}(\mathbf{y}(\tau), \dot{\mathbf{y}}(\tau), \tau)=0 .
$$

The nonlinear mode can be sought in the form of a decaying multiharmonic vibration, which has the following form:

$$
\mathbf{y}(\tau)=\operatorname{Re}\left(\sum_{k=1}^{N_{h}} \mathbf{Y}^{k} e^{k \lambda \tau}\right)
$$

where $\mathrm{Y}^{k}$ is a complex vector containing the coefficients associated to the $k$ th harmonic for all DOFs, which represents the nonlinear modal shape of the substructure, $\lambda$ is the complex eigenvalue associated with the nonlinear mode, and $\operatorname{Re}(\bullet)$ represents the operation for obtaining the real part. The same procedure is then applied for obtaining the nonlinear force $\mathbf{g}_{\mathrm{nl}}(\mathbf{y}, \dot{\mathbf{y}}, \tau)$, and they can be expressed in terms of a truncated Fourier series:

$$
\mathbf{g}_{\mathrm{nl}}(\mathbf{y}(\tau), \dot{\mathbf{y}}(\tau), \tau)=\operatorname{Re}\left(\sum_{k=1}^{N_{h}} \mathbf{G}_{\mathrm{nl}}^{k} e^{k \lambda \tau}\right)
$$

where $\mathbf{G}_{\mathrm{nl}}^{k}$ is the complex vector containing the coefficients of the nonlinear force. By substituting (10) and (11) into (9), the nonlinear algebraic equations can be obtained using the Galerkin procedure:

$$
\mathbf{H}(\lambda) \cdot \mathbf{Y}(\lambda)+\mathbf{G}_{\mathrm{nl}}(\lambda, \mathbf{Y})=0,
$$

where $\mathbf{H}(\lambda)$ is the dynamic stiffness matrix, and we can calculate the coefficient vectors of the nonlinear force using the AFT method and ignore the decrease in $\mathbf{Y}$ over one period.

To solve (12), two more equations should be added to make the equations complete. The first is the correction equation in the correction step of the arc-length continuation procedure, and the other one can be obtained by imposing the phase of one harmonic of a DOF. When solving the equation, the initial condition can be determined from the underlying linear mode of the system. Using this procedure, the nonlinear modal shape $\mathbf{Y}$, damped natural frequency, which is the imaginary part of $\lambda$, and modal damping ratio, which

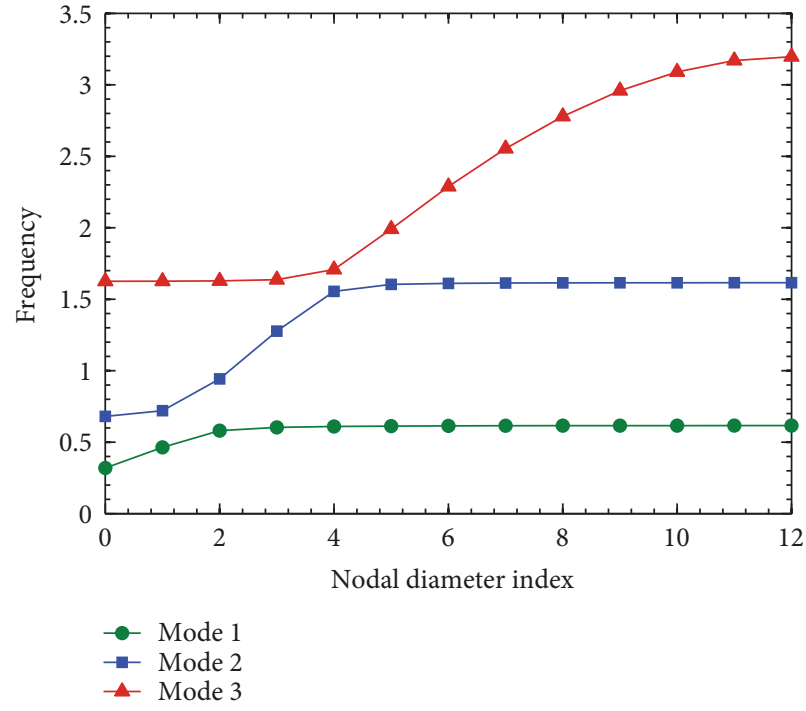

FIGURE 5: Frequency/nodal diameter index (NDI) map of the blisk.

is the real part of $\lambda$, can be obtained by solving these equations. More details on the solving procedure can be found in literature [34, 37].

3.3. Nonlinear Complex Modal Properties. Because the mass $\delta_{\text {me }}$ and stiffness $\gamma_{\mathrm{e}}$ of a piezo-patch may influence the dynamic behaviour of the blisk, they are considered in the calculation. The mass $\delta_{\text {me }}$ is taken as $5 \%$ of the blade-root mass $\delta_{\mathrm{mb}}$, and the stiffness $\gamma_{\mathrm{e}}$ is taken as $5 \%$ of $\gamma_{\mathrm{b}}$. The main nondimensional parameters used are listed in Table 1. $\gamma_{\mathrm{b}}=1$ in the table represents the configuration where the stiffness of blade and that of disk are close. In this case, the blade-diskcoupling vibration would occur.

With this set of parameters, the natural frequencies of the linear blisk without the SSDNC are calculated, and the results are presented schematically as a function of the nodal diameter index (NDI) of the blisk modes in Figure 5. There are three frequency loci in the figure as the lumped parameter model consists of three DOFs per sector. The largest NDI is $N / 2$ if the blade number $N$ is even, while it is $(N-1) / 2$ if $N$ is odd. The phenomenon wherein two loci come close gradually at first and then separate with the increase in the NDI is known as the frequency veering. The region where the frequency loci are closest is called the frequency-veering region. The NDI nearest to the frequency-veering region is 1,2 , or 4 for this analysis model. A previous study [1, 38] has proven that the forced response of the blisk is more sensitive to mistuning in the frequency-veering region; therefore, the mistuning analysis presented in the following section will be performed in this region.

When the capacitance ratio $\chi_{c}$ is 0.85 , the modal frequencies and modal damping ratios of the nonlinear substructure 


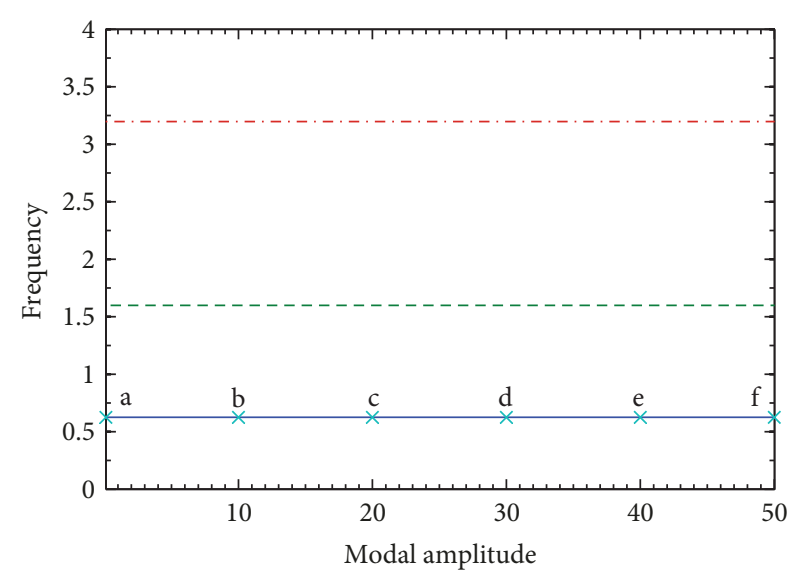

(a) Natural frequencies of the substructure

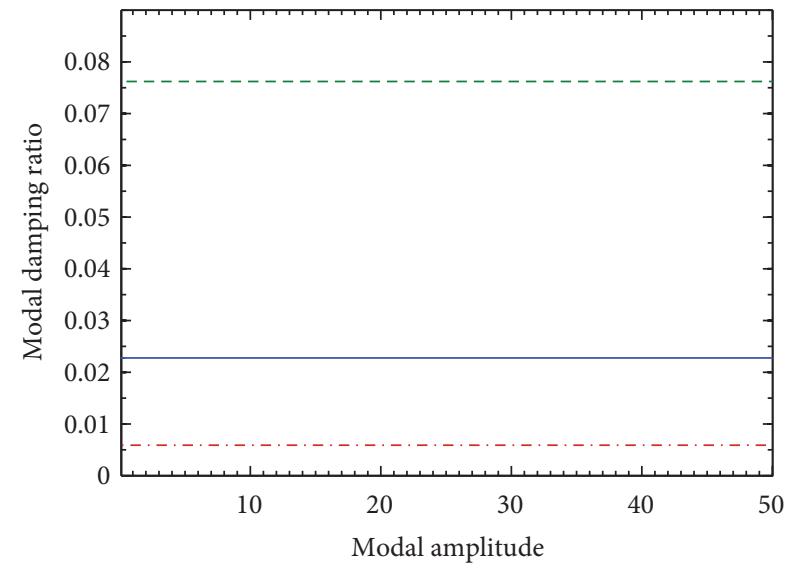

(b) Modal damping ratios of the substructure

FIgURE 6: Modal information of the nonlinear substructure.
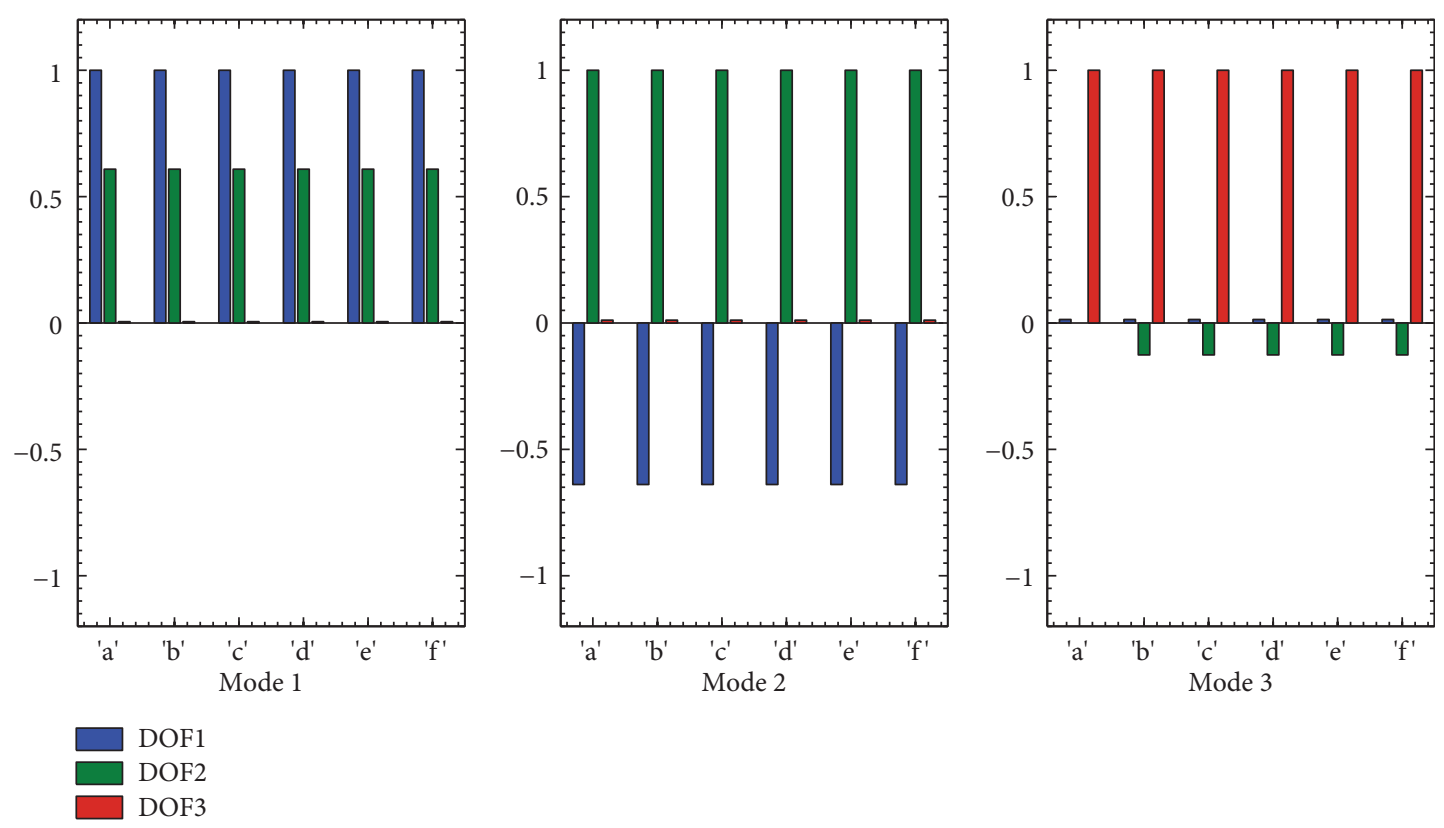

FIgURE 7: Modal shapes of the substructure.

corresponding to different modal amplitudes are obtained following the procedure given in Section 3.2 when the boundary DOFs are fixed. The variation tendency of the damped natural frequencies of the three modes is shown in Figure 6(a), and that of the damping ratios is shown in Figure 6(b). From these figures, we can observe that both the frequencies and damping ratios of the substructures remain almost unchanged with an increasing modal amplitude. This behaviour is different from general nonlinear systems (such as the dry-friction system [37]), in which the modal properties vary with the modal amplitude.

The modal shapes of several cases indicated with the symbol " $x$ " in Figure 6(a) are shown in Figure 7. These figures show that the mode shapes of the system do not change with the modal amplitude. These results indicate that although the SSDNC is an essential nonlinear element, the nonlinearity of the electromechanical system associated with it is less apparent. This characteristic of the SSDNC could be used to reduce the calculation cost of the nonlinear system. Here, it should be noted that although the mode shapes and natural frequencies do not change with respect to the nonlinear modal amplitude, the forced response of the blisk with SSDNC cannot be solved directly by the linear analysis because the equivalent modal stiffness and modal damping ratio of the SSDNC are unknown. A nonlinear analysis is still required in this case to get those equivalent parameters.

\section{Nonlinear Component Modal Synthesis Method}

Although the MHBM-AFT method can reduce the computation time significantly, it is still a challenge to carry out statistical analysis of a nonlinear blisk with random mistuning. It is known that the component modal synthesis 
(CMS) method is an effective reduction algorithm for analysing complex linear systems. Considering the efficiency of the CMS method, Krack et al. [39] and Joannin et al. [37, 40] extended the method to nonlinear systems such as the dryfriction system; this method is referred to as the nonlinear component complex modal synthesis method. In their analysis, iterative calculation was necessary because the modal information of the nonlinear system is dependent on the modal amplitude. The nonlinear modal analysis presented in Section 3 indicates that the modal information of the SSDNC system remains almost unchanged with the increasing modal amplitude. Inspired by this result, we propose using the nonlinear component modal synthesis method to obtain the forced response of the blisk with the SSDNC in the frequency domain, but omitting the iteration procedure (note: the iteration procedure is still needed in the process of calculating the nonlinear modal information), which will increase the computational efficiency significantly. To verify this idea, in this section, the procedure of the nonlinear modal synthesis method is introduced and its computational precision and efficiency will be compared with that of the MHBM-AFT.

4.1. Nonlinear Complex Modal Synthesis. Similar to the classical CMS method with a fixed interface, all the DOFs of the nonlinear substructure can be partitioned into internal DOFs (subscript "i") and boundary DOFs (subscript "b"). If no external excitation force is applied on the boundary DOFs, (9) can be rewritten as follows:

$$
\begin{aligned}
\lambda^{2}\left[\begin{array}{cc}
\mathbf{M}_{\mathrm{ii}} & \mathbf{M}_{\mathrm{ib}} \\
\mathbf{M}_{\mathrm{bi}} & \mathbf{M}_{\mathrm{bb}}
\end{array}\right]\left(\begin{array}{c}
\ddot{\mathbf{y}}_{\mathrm{i}} \\
\ddot{\mathbf{y}}_{\mathrm{b}}
\end{array}\right)+\lambda\left[\begin{array}{cc}
\mathbf{C}_{\mathrm{ii}} & \mathbf{C}_{\mathrm{ib}} \\
\mathbf{C}_{\mathrm{bi}} & \mathbf{C}_{\mathrm{bb}}
\end{array}\right]\left(\begin{array}{c}
\dot{\mathbf{y}}_{\mathrm{i}} \\
\dot{\mathbf{y}}_{\mathrm{b}}
\end{array}\right) \\
+\left[\begin{array}{cc}
\mathbf{K}_{\mathrm{ii}} & \mathbf{K}_{\mathrm{ib}} \\
\mathbf{K}_{\mathrm{bi}} & \mathbf{K}_{\mathrm{bb}}
\end{array}\right]\left(\begin{array}{c}
\mathbf{y}_{\mathrm{i}} \\
\mathbf{y}_{\mathrm{b}}
\end{array}\right)+\left(\begin{array}{c}
\mathbf{g}_{\mathrm{nl}}\left(\mathbf{y}_{\mathrm{i}}, \dot{\mathbf{y}}_{\mathrm{i}}, \tau\right) \\
0
\end{array}\right)=\left(\begin{array}{c}
\mathbf{g}_{\mathrm{i}}(\tau) \\
0
\end{array}\right) .
\end{aligned}
$$

When the boundary DOFs are fixed $\left(\mathbf{y}_{\mathrm{b}}=0\right)$ and the external excitation forces are ignored, the dynamic equations of the nonlinear substructure can be obtained from (13):

$$
\lambda^{2} \mathbf{M}_{\mathrm{ii}} \ddot{\mathrm{y}}_{\mathrm{i}}(\tau)+\lambda \mathbf{C}_{\mathrm{ii}} \dot{\mathbf{y}}_{\mathrm{i}}(\tau)+\mathbf{K}_{\mathrm{ii}} \mathbf{y}_{\mathrm{i}}(\tau)+\mathbf{g}_{\mathrm{nl}}\left(\mathbf{y}_{\mathrm{i}}(\tau), \dot{\mathbf{y}}_{\mathrm{i}}(\tau), \tau\right)=0 .
$$

When the procedure described in Section 3.2 is applied, the nonlinear modal-shape matrix $\boldsymbol{\Phi}_{\mathrm{i}}$ and eigenvalue matrix $\Lambda_{\mathrm{i}}$ (which is a diagonal matrix containing the eigenvalues) can be solved. The static mode-shape matrix $\boldsymbol{\Phi}_{\mathrm{b}}$ can be obtained using the stiffness matrix of the substructure:

$$
\Phi_{\mathrm{b}}=-\mathbf{K}_{\mathrm{ii}}^{-1} \cdot \mathbf{K}_{\mathrm{ib}} .
$$

Then the physical coordination (represented by $\mathbf{y}=$ $\left.\left[\mathbf{y}_{\mathrm{i}}, \mathbf{y}_{b}\right]^{T}\right)$ can be transformed into the modal coordination (represented by $\mathbf{p}=\left[\mathbf{p}_{\mathrm{i}}, \mathbf{p}_{\mathrm{b}}\right]^{T}$ ) using matrix $\boldsymbol{\Phi}$ :

$$
\mathbf{y}=\boldsymbol{\Phi} \cdot \mathbf{p} .
$$

The specific form of (16) is as follows:

$$
\left(\begin{array}{c}
\mathbf{y}_{\mathrm{i}} \\
\mathbf{y}_{\mathrm{b}}
\end{array}\right)=\left[\begin{array}{cc}
\Phi_{\mathrm{i}} & \boldsymbol{\Phi}_{\mathrm{b}} \\
\mathbf{0} & \mathbf{I}_{\mathrm{b}}
\end{array}\right]\left(\begin{array}{l}
\mathbf{p}_{\mathrm{i}} \\
\mathbf{p}_{\mathrm{b}}
\end{array}\right),
$$

Combining (12) and (17), we can obtain the nonlinear force using the nonlinear modal information as follows:

$$
\mathbf{G}_{\mathrm{nl}}=-\left(\mathbf{M}_{\mathrm{ii}} \Lambda_{\mathrm{i}}^{2}+\mathbf{C}_{\mathrm{ii}} \Lambda_{\mathrm{i}}+\mathbf{K}_{\mathrm{ii}}\right) \cdot \Phi_{\mathrm{i}} \cdot \mathbf{p}_{\mathrm{i}} \cdot
$$

Then the dynamic equations of substructure $s$ in the frequency domain can be obtained:

$$
\mathbf{H}^{s} \cdot \mathbf{p}^{s}=\mathbf{G}^{s}
$$

where $s=1,2, \ldots, N$. The specific form of (19) is as follows:

$$
\left[\begin{array}{cc}
\mathbf{H}_{\mathrm{ii}} \cdot \boldsymbol{\Phi}_{\mathrm{i}}-\mathbf{Q}_{\mathrm{ii}} & \mathbf{H}_{\mathrm{ii}} \cdot \Phi_{\mathrm{b}}+\mathbf{H}_{\mathrm{ib}} \\
\mathbf{H}_{\mathrm{bi}} \cdot \boldsymbol{\Phi}_{\mathrm{i}} & \mathbf{H}_{\mathrm{bi}} \cdot \Phi_{\mathrm{b}}+\mathbf{H}_{\mathrm{bb}}
\end{array}\right]\left(\begin{array}{c}
\mathbf{p}_{\mathrm{i}} \\
\mathbf{p}_{\mathrm{b}}
\end{array}\right)=\left(\begin{array}{c}
\mathbf{G}_{\mathrm{i}} \\
\mathbf{0}
\end{array}\right),
$$

Here, the submatrices have the following forms:

$$
\begin{aligned}
\mathbf{H}_{\mathrm{ii}} & =-\lambda^{2} \mathbf{M}_{\mathrm{ii}}+\lambda \mathbf{C}_{\mathrm{ii}}+\mathbf{K}_{\mathrm{ii}}, \\
\mathbf{H}_{\mathrm{ib}} & =-\lambda^{2} \mathbf{M}_{\mathrm{ib}}+\lambda \mathbf{C}_{\mathrm{ib}}+\mathbf{K}_{\mathrm{ib}}, \\
\mathbf{H}_{\mathrm{bi}} & =-\lambda^{2} \mathbf{M}_{\mathrm{bi}}+\lambda \mathbf{C}_{\mathrm{bi}}+\mathbf{K}_{\mathrm{bi}}, \\
\mathbf{H}_{\mathrm{bb}} & =-\lambda^{2} \mathbf{M}_{\mathrm{bb}}+\lambda \mathbf{C}_{\mathrm{bb}}+\mathbf{K}_{\mathrm{bb}}, \\
\mathbf{Q}_{\mathrm{ii}} & =\mathbf{M}_{\mathrm{ii}} \boldsymbol{\Phi}_{\mathrm{i}} \Lambda_{\mathrm{i}}^{2}+\mathbf{C}_{\mathrm{ii}} \boldsymbol{\Phi}_{\mathrm{i}} \Lambda_{\mathrm{i}}+\mathbf{K}_{\mathrm{ii}} \boldsymbol{\Phi}_{\mathrm{i}} .
\end{aligned}
$$

Because the tuned blisk is a typical cyclic periodic structure, the modal coordinates of the boundary DOFs can be partitioned into $\mathbf{p}_{\mathrm{b}}=\left[\mathbf{p}_{\mathrm{b}, \mathrm{r}}^{s}, \mathbf{p}_{\mathrm{b}, \mathrm{l}}^{s}\right]^{T}$, and the following relationship can be obtained:

$$
\begin{aligned}
& \mathbf{p}_{\mathrm{b}, \mathrm{r}}^{s}=\mathbf{p}_{\mathrm{b}, \mathrm{l}}^{s+1}, \\
& \mathbf{p}_{\mathrm{b}, \mathrm{l}}^{s}=\mathbf{p}_{\mathrm{b}, \mathrm{r}}^{s-1} .
\end{aligned}
$$

Using the relationship in (22), we can obtain the transformation matrix $\Psi$, which can assemble all the substructures to build the entire nonlinear blisk system. The circulant block matrix $\Psi$ has the following form:

$$
\boldsymbol{\Psi}=\left[\begin{array}{ccccc}
\mathbf{I}_{1} & 0 & 0 & \cdots & \mathbf{I}_{0} \\
\mathbf{I}_{0} & \mathbf{I}_{1} & 0 & \cdots & 0 \\
0 & \mathbf{I}_{0} & \mathbf{I}_{1} & \cdots & 0 \\
\vdots & \vdots & \vdots & \ddots & \vdots \\
0 & 0 & 0 & \cdots & \mathbf{I}_{1}
\end{array}\right],
$$

Here, the submatrices have the following forms: 


$$
\begin{aligned}
\mathbf{I}_{1} & =\left[\begin{array}{llll}
1 & 0 & 0 & 0 \\
0 & 1 & 0 & 0 \\
0 & 0 & 1 & 0 \\
0 & 0 & 0 & 1 \\
0 & 0 & 0 & 0
\end{array}\right], \\
\mathbf{I}_{0} & =\left[\begin{array}{llll}
0 & 0 & 0 & 0 \\
0 & 0 & 0 & 0 \\
0 & 0 & 0 & 0 \\
0 & 0 & 0 & 0 \\
0 & 0 & 0 & 1
\end{array}\right] .
\end{aligned}
$$

The dynamic equations of the blisk with the SSDNC in the frequency domain in modal coordination are as follows:

$$
\tilde{\mathbf{H}} \cdot \tilde{\mathbf{p}}=\tilde{\mathbf{G}},
$$

where the matrix $\tilde{\mathbf{H}}$ and the vectors $\tilde{\mathbf{p}}$ and $\tilde{\mathbf{G}}$ can be obtained using the transformation matrix $\Psi$ :

$$
\begin{aligned}
\tilde{\mathbf{H}} & =\boldsymbol{\Psi}^{T} \mathbf{H} \boldsymbol{\Psi}, \\
\tilde{\mathbf{p}} & =\boldsymbol{\Psi}^{T} \mathbf{p}, \\
\tilde{\mathbf{G}} & =\boldsymbol{\Psi}^{T} \mathbf{G} .
\end{aligned}
$$

Here, the matrix $\mathbf{H}$ and the vectors $\mathbf{p}$ and $\mathbf{G}$ have the following forms:

$$
\begin{aligned}
& \mathbf{H}=\left[\begin{array}{llll}
\mathbf{H}^{1} & & & \\
& \mathbf{H}^{2} & & \\
& & \ddots & \\
& & & \mathbf{H}^{N}
\end{array}\right], \\
& \mathbf{p}=\left[\mathbf{p}^{1}, \mathbf{p}^{2}, \ldots, \mathbf{p}^{N}\right]^{T}, \\
& \mathbf{G}=\left[\mathbf{G}^{1}, \mathbf{G}^{2}, \ldots, \mathbf{G}^{N}\right]^{T} .
\end{aligned}
$$

The rotating blisk in aeroengines is subjected to the traveling wave excitation induced by upstream vanes and the rotation itself, and the exciting force has the same magnitude but different phases in each sector. It is usually called engineorder excitation. With $E$ denoting the engine order, the excitation of substructure $s$ has the following expression:

$$
\mathbf{g}^{s}(\tau)=\left[\mathbf{g}_{1}(\boldsymbol{\tau}), \boldsymbol{\delta}_{\mathrm{fb}} \cdot \mathbf{g}_{1}(\boldsymbol{\tau}), \boldsymbol{\delta}_{\mathrm{fd}} \cdot \mathbf{g}_{1}(\boldsymbol{\tau})\right]^{T} \otimes e^{i \cdot(s-1) \cdot E \cdot \theta},
$$

where $\left[\mathbf{g}_{1}(\boldsymbol{\tau}), \boldsymbol{\delta}_{\mathrm{fb}} \cdot \mathbf{g}_{1}(\boldsymbol{\tau}), \boldsymbol{\delta}_{\mathrm{fd}} \cdot \mathbf{g}_{1}(\boldsymbol{\tau})\right]^{T}$ is the excitation vector applied to the reference substructure. The symbol $\otimes$ denotes the Kronecker tensor product, $i$ is the imaginary unit, and $\theta=2 \pi / N$ is the phase difference between adjacent blades. When the vector $\tilde{\mathbf{p}}$ is obtained, the forced response of the blisk can be obtained by the inverse coordination transformation based on (17) and (26).

This procedure is referred to as the nonlinear component modal synthesis method (NCMS). When the procedure is

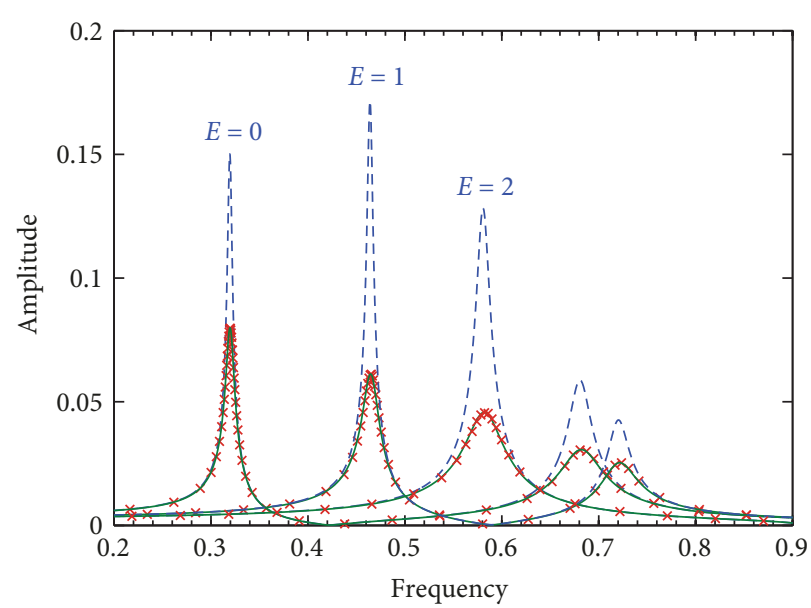

FIGURE 8: Forced response of the tuned blisk with SSDNC (solid curves: NCMS method; curves with symbol " $x$ ": MHBM-AFT) and that without SSDNC (dash curves).

TABLE 2: Comparison of computation time for the tuned blisk with SSDNC.

\begin{tabular}{lccc}
\hline Method & Frequency steps & Total time & Time percentage \\
\hline NCMS & 1900 & $15.591 \mathrm{~s}$ & $0.72 \%$ \\
MHBM-AFT & 129 & $2136.10 \mathrm{~s}$ & $100 \%$ \\
\hline
\end{tabular}

used, a nonlinear modal analysis should be performed first to obtain the nonlinear modal information of one substructure. However, because the calculation is limited to one substructure, the calculating scale will be much smaller. It should be noted that although a lumped parameter model with only three DOFs in each sector is used in the investigation, the high-fidelity model of the blisk can also be used in the analysis, and the computation time will be considerably reduced.

\subsection{Precision and Efficiency of NCMS Method}

4.2.1. Tuned Blisk with SSDNC. When the capacitance ratio $\chi_{c}$ is taken as 0.85 , the forced responses of the tuned blisk with the SSDNC in the frequency domain under engineorder excitations, obtained using NCMS method and MHBM-AFT, respectively, are compared in Figure 8. As a reference, the forced response of the blisk without the SSDNC is also shown in Figure 8. From the figure, we can see that apart from the effectiveness of the SSDNC in suppressing vibration, the response curve obtained using NCMS method and that using MHBM-AFT coincides well, which means that the NCMS method is precise enough to calculate the forced response of the tuned blisk with the SSDNC.

The total computation time of the NCMS method and that of the MHBM-AFT are listed and compared in Table 2. When the engine order is 2 , the total computation time for the forced response using the NCMS method is approximately $15.591 \mathrm{~s}$. It consists of the time for the modal analysis of substructures and the time for calculating the response via nonlinear modal synthesis. Because the blisk is tuned, all the nonlinear substructures are the same, so the 
TABLE 3: Mistuned sample of the blade stiffness.

\begin{tabular}{lcccccccc}
\hline$j$ & 1 & 2 & 3 & 4 & 5 & 6 & 7 & 8 \\
$\delta \gamma_{\mathrm{b}, j}$ & 0.02445 & 0.05174 & 0.03634 & -0.01517 & 0.01469 & -0.03936 & 0.04442 & -0.05735 \\
\hline$j$ & 9 & 10 & 11 & 12 & 13 & 14 & 15 & 16 \\
$\delta \gamma_{\mathrm{b}, j}$ & -0.05344 & -0.04047 & -0.1472 & 0.07192 & 0.01626 & -0.03775 & 0.06852 & -0.08558 \\
\hline$j$ & 17 & 18 & 19 & 20 & 21 & 22 & 23 & 24 \\
$\delta \gamma_{\mathrm{b}, j}$ & -0.00511 & -0.01207 & 0.01596 & 0.01564 & -0.04324 & -0.00150 & -0.00824 & 0.03139 \\
\hline
\end{tabular}

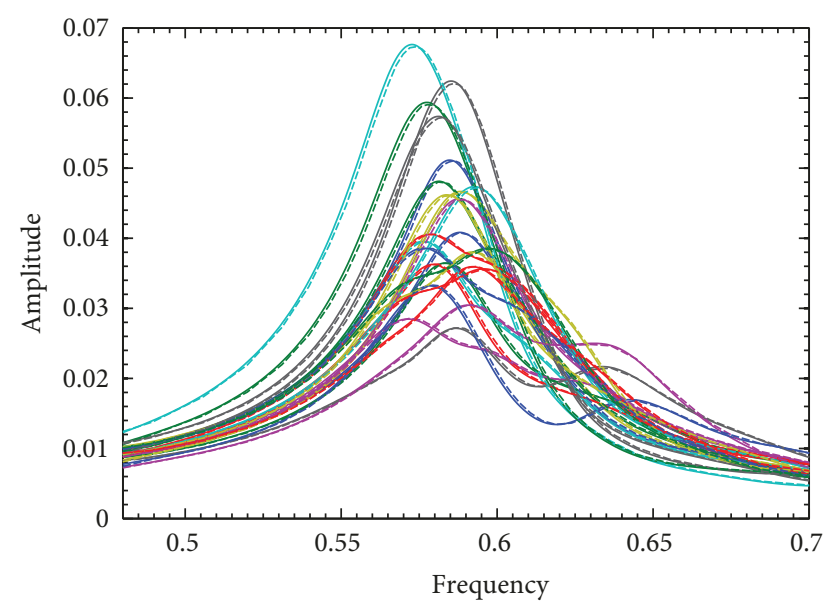

FIGURE 9: Forced response of the mistuned blisk with SSDNC (solid curves: NCMS method; dash curves: MHBM-AFT).

modal information needs to be calculated only once. In addition, because the modal information of the substructure remains unchanged with the increasing modal amplitude (as shown in Figures 6 and 7), we omit the iteration in calculating the forced response. As a result, the computation time for obtaining the nonlinear modal information is small. The total computation time of the MHBM-AFT is approximately $2136.10 \mathrm{~s}$; that is to say, the computation time of the NCMS method is only approximately $0.72 \%$ of that of the MHBMAFT. The result shows that the NCMS method can reduce the calculation time significantly.

4.2.2. Mistuned Blisk with SSDNC. It is supposed that random mistuning of the blisk occurred in the stiffness of the blade bodies and blade roots, and the nondimensional mistuned stiffness in sector $j$ is defined as $\delta \gamma_{\mathrm{b} 1, j}=\delta \gamma_{\mathrm{b} 2, j}=\delta \gamma_{\mathrm{b}, j}$. A group of random mistuning samples of the blade stiffness used in the calculation is listed in Table 3. It follows a normal distribution with a mean value of 0 and a standard deviation (called "mistuned level" in the following text and denoted by $\Delta \gamma_{\mathrm{b}}$ ) of $5 \%$. When the capacitance ratio $\chi_{\mathrm{c}}$ is taken as 0.85 , the forced response of the mistuned blisk with the SSDNC obtained using the NCMS method and MHBM-AFT, respectively, is compared in Figure $9(E=2)$. It can be seen that the amplitude of the forced response of blades is different from one sector to another rather than being the same, as observed in the tuned blisk. The forced responses of different blades

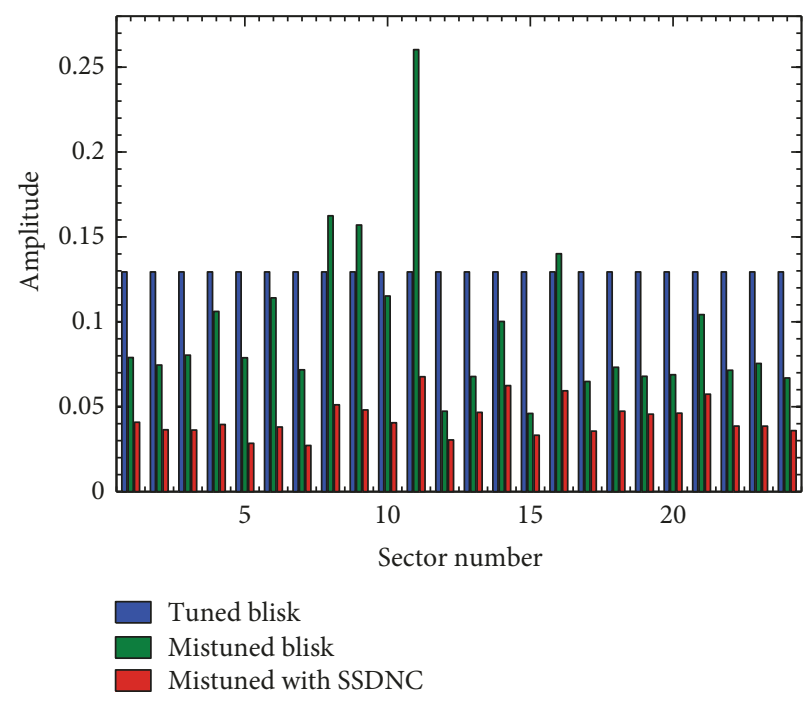

FIGURE 10: Highest response amplitude of different blades in the mistuned blisk.

obtained by the NCMS method and that obtained by MHBM-AFT coincide with each other well (the maximum calculation error of the forced response of different blades is approximately $0.48 \%$ ), which means that the NCMS method is precise enough to calculate the forced response even in the mistuned condition.

The maximum response amplitude of the different blades in the mistuned blisk with and without the SSDNC is shown in Figure 10. According to the figure, some blades could have significantly larger forced responses than the tuned one (e.g., the nondimensional amplitude of the blade body in sector 11 is approximately $94.92 \%$ larger than that of the tuned blisk). This phenomenon is known as amplitude magnification. However, with the SSDNC, not only is the amplitude magnification suppressed but also the forced response of different blades is much smaller than that of the tuned blisk.

When the blisk is mistuned, all its nonlinear substructures are different from each other. To obtain the forced response of the entire blisk, the modal information of all the substructures should be calculated. Therefore, the total computation time in the mistuned condition is much larger than that in the tuned condition. A comparison of the computation time of the NCMS method and that of the MHBMAFT is presented in Table 4. The NCMS method, taking advantage of modal properties of the system, considerably 
TABLE 4: Comparison of computation time of the tuned blisk with SSDNC.

\begin{tabular}{lccc}
\hline Method & Frequency steps & Total time & Time percentage \\
\hline NCMS & 1900 & $65.816 \mathrm{~s}$ & $2.72 \%$ \\
MHBM-AFT & 130 & $2415.765 \mathrm{~s}$ & $100 \%$ \\
\hline
\end{tabular}

reduces the calculation time. This result implicates that for a high-fidelity model of the blisk, the calculation time of the NCMS method could be acceptable.

\section{Statistical Analysis}

The advantage of using the NCMS method for analysing a system with the SSDNC has been verified in Section 4. In this section, it is used to perform a statistical analysis of the random mistuned blisk with the SSDNC.

5.1. Vibration-Suppression Performance of SSDNC. The amplitude magnification factor (AMF) is often used to measure the response amplification of the mistuned blisk [1]. Below, we define 2 AMFs to evaluate the damping effect of the SSDNC in the mistuned blisk.

$$
\begin{aligned}
\mathrm{AMF}_{1} & =\frac{A_{\mathrm{m}}}{A_{\mathrm{t}}}, \\
\mathrm{AMF}_{2} & =\frac{A_{\mathrm{m}, \mathrm{SSDNC}}}{A_{\mathrm{t}}} .
\end{aligned}
$$

Here, $A_{\mathrm{t}}$ is the response amplitude of the blades in the tuned blisk without the SSDNC, and $A_{\mathrm{m}}$ and $A_{\mathrm{m}, \mathrm{SSDNC}}$ are the highest amplitudes of all the blades in the mistuned blisk with and without the SSDNC, respectively. $\mathrm{AMF}_{1}$ is used to evaluate the effect of mistuning on a blisk without the SSDNC, and $\mathrm{AMF}_{2}$ is used to evaluate the same with the SSDNC.

Following the procedure of statistical analysis, we can obtain the estimations of $\mathrm{AMF}_{1}$ and $\mathrm{AMF}_{2}$ with a $99.9 \%$ confidence interval when the blisk is excited by different engine orders. Each estimation is based on 500 samples of random mistuning, which follow a normal distribution. The mean of the samples is 0 , and the standard deviation is taken from 0 to $15 \%$ with an increment of $0.5 \%$. The estimations of both $\mathrm{AMF}_{1}$ and $\mathrm{AMF}_{2}$ corresponding to different engine orders and mistuning levels are compared in Figure 11. From a statistical viewpoint, the forced response of the blisk is amplified significantly (the statistical value of $\mathrm{AMF}_{1}$ is 1.785 when $E$ is 2 and mistuning level is 7\%) because of random mistuning. When the SSDNC is introduced into the system, $\mathrm{AMF}_{2}$ is much smaller than $\mathrm{AMF}_{1}$. Figure 12 gives the probability density function (PDF) of $\mathrm{AMF}_{1}$ and $\mathrm{AMF}_{2}$ based on 500 samples when the engine order is 2 and the mistuning level is $7 \%$. As observed in the figure, $\mathrm{AMF}_{1}$ varies from 1.3679 to 2.2234 with a mean of approximately 1.785 . $\mathrm{AMF}_{2}$ varies from 0.2973 to 0.4063 with a mean of approximately 0.3515 , which is much smaller than that of $\mathrm{AMF}_{1}$. Therefore, from a statistical viewpoint, we can conclude that the SSDNC can reduce the amplified response of the mistuned blisk;

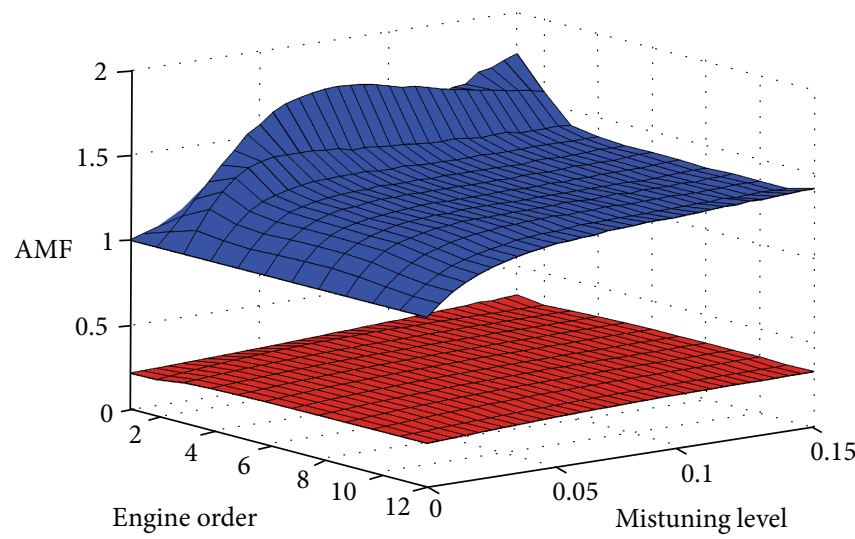

FIGURE 11: Estimations of AMF for the mistuned blisk with (red) and without (blue) SSDNC.

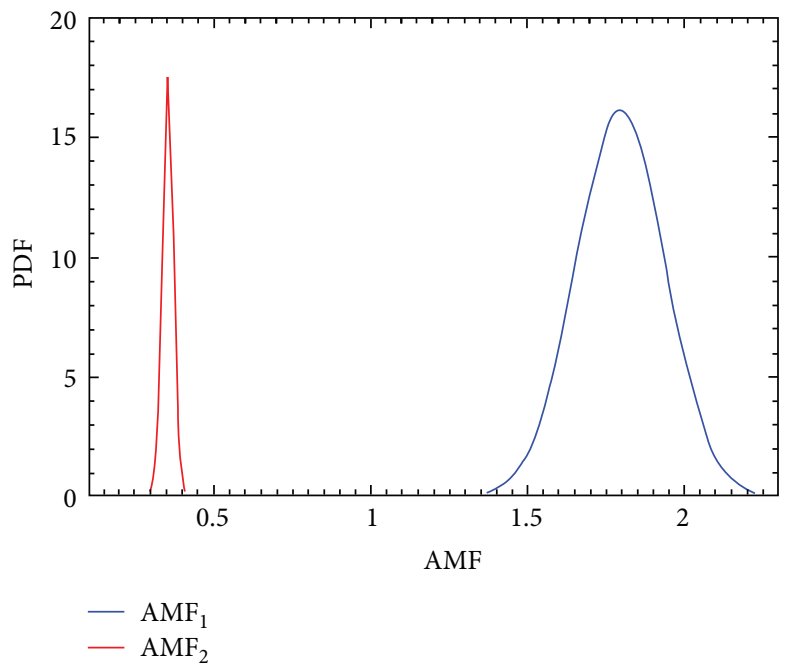

Figure 12: Probability density function of $\operatorname{AMF}\left(E=2, \triangle \gamma_{\mathrm{b}}=7 \%\right)$.

further, it can completely eliminate the response amplification of the blisk even in the case with a mistuning level as large as $15 \%$ (all AMFs < 1 ).

5.2. Delocalization Performance of SSDNC. The difference in the vibration amplitude of the blades induced by mistuning is usually measured through the response localization factor (RLF) [41, 42]. For the mistuned blisk with or without the SSDNC, the RLFs are defined as below:

$$
\begin{aligned}
\mathrm{RLF}_{1} & =\frac{A_{\mathrm{m}}^{\mathrm{max}}-A_{\mathrm{m}}^{\min }}{A_{\mathrm{t}}} \\
\text { or } \mathrm{RLF}_{2} & =\frac{A_{\mathrm{m}, \mathrm{SSDNC}}^{\mathrm{max}}-A_{\mathrm{m}, \mathrm{SSDNC}}^{\min }}{A_{\mathrm{t}, \mathrm{SSDNC}}} .
\end{aligned}
$$

Here, $A_{\mathrm{m}}^{\max }$ and $A_{\mathrm{m}}^{\min }$ are, respectively, the highest and lowest vibration amplitudes of the blades in the mistuned blisk without the SSDNC, and $A_{\mathrm{m}, S S D N C}^{\max }$ and $A_{\mathrm{m}, \mathrm{SSDNC}}^{\mathrm{min}}$ are those in the mistuned blisk with the SSDNC. $\mathrm{RLF}_{1}$ is used to evaluate the localization level of the mistuned blisk 


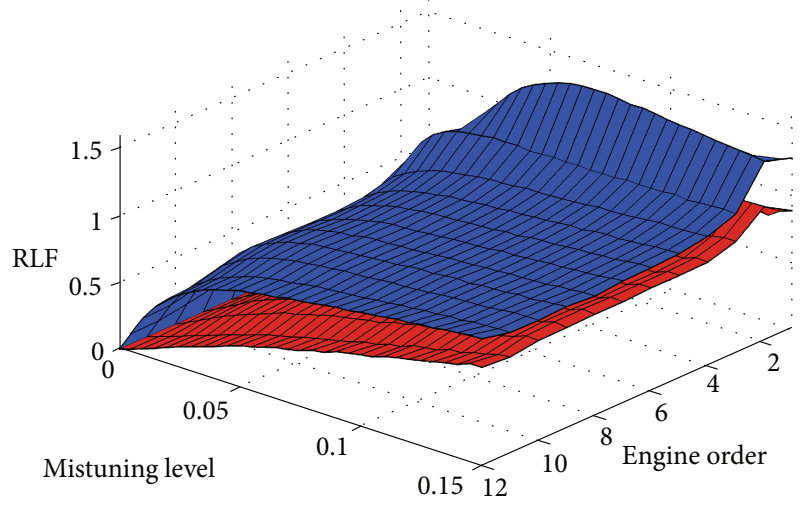

FIGURE 13: Response localization factor of the mistuned blisk with (red) and without (blue) SSDNC.

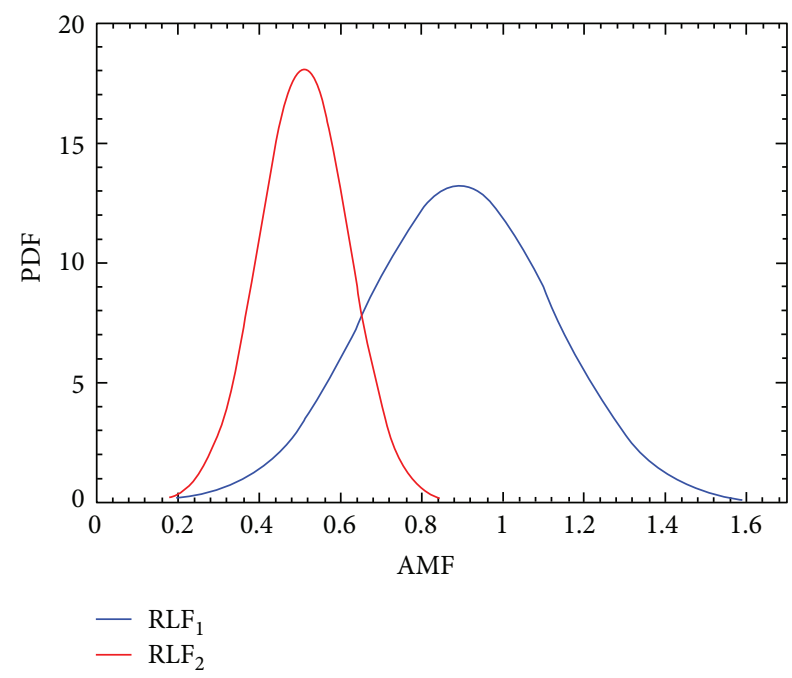

Figure 14: Probability density function of RLF $\left(E=2, \triangle \gamma_{\mathrm{b}}=7 \%\right)$.

without the SSDNC, and $\mathrm{RLF}_{2}$ is used to evaluate that with the SSDNC.

The estimated $\mathrm{RLF}_{1}$ and $\mathrm{RLF}_{2}$ of the blisk with a $99.9 \%$ confidence interval corresponding to excitations of different engine orders and mistuning levels are compared in Figure 13. It can be seen that, for all the conditions, the statistical value of $R L F_{2}$ is lower than that of $R L F_{1}$. Figure 14 gives the PDF of RLF ${ }_{1}$ and $R L F_{2}$ based on 500 samples when the engine order is 2 and the mistuning level is $7 \%$. From the figure, the mean value of RLF is reduced from 0.892 to 0.507 , owing to the SSDNC. From the viewpoint of statistical analysis, we can conclude that the SSDNC is capable of reducing the difference in the forced response amplitude between blades under engine-order excitations.

5.3. Mistuning Sensitivity of SSDNC. To evaluate the sensitivity of the blisk with the SSDNC to mistuning, we define A $\mathrm{MF}_{3}$ as below:

$$
\mathrm{AMF}_{3}=\frac{A_{\mathrm{m}, \mathrm{SSDNC}}}{A_{\mathrm{t}, \mathrm{SSDNC}}} .
$$

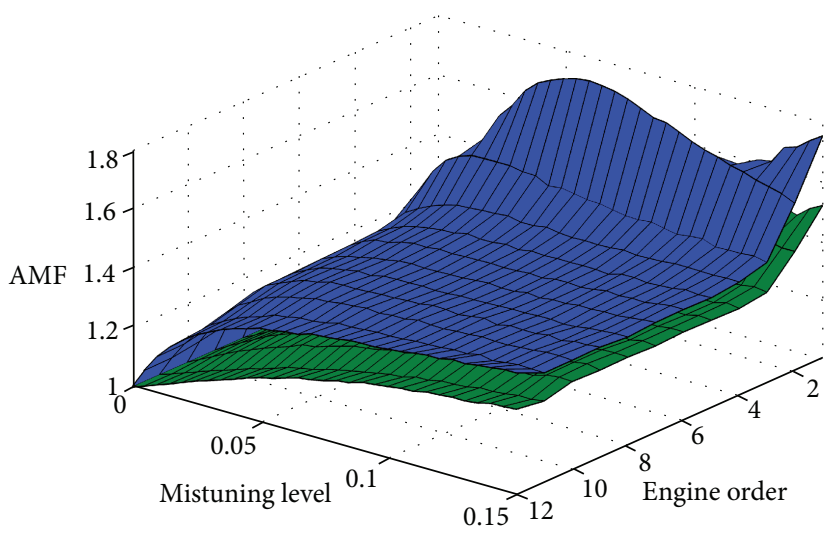

FIGURE 15: Sensitivity to engine orders and mistuning levels.

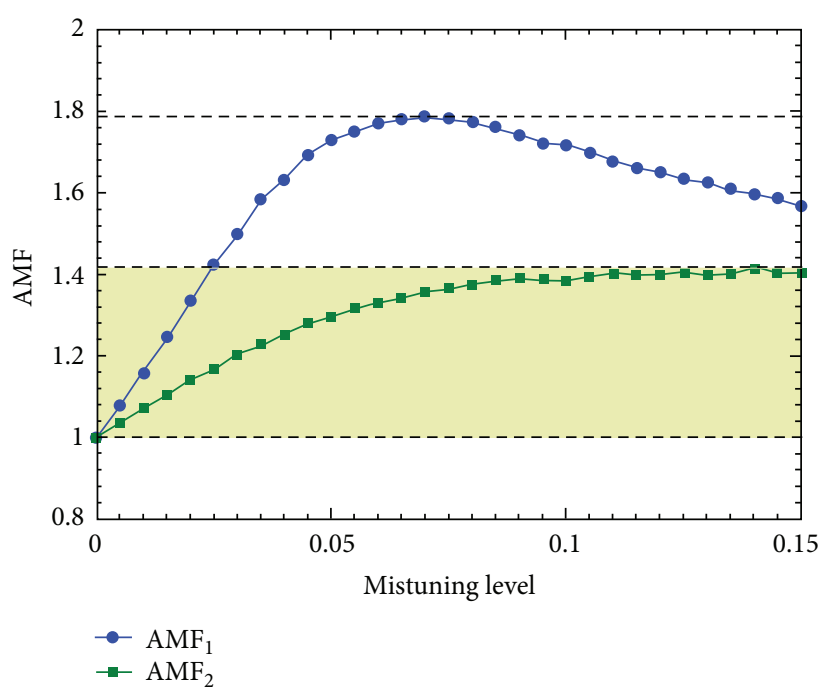

FIgURE 16: Sensitivity to mistuning levels $(E=2)$.

Here, $A_{\mathrm{t}, \mathrm{SSDNC}}$ and $A_{\mathrm{m}, \mathrm{SSDNC}}$ are the highest amplitudes of the blades in the tuned and mistuned blisk with the SSDNC, respectively. The estimation of $\mathrm{AMF}_{3}$ for the blisk with a $99.9 \%$ confidence interval is compared with that of $\mathrm{AMF}_{1}$ (the effect of mistuning on a blisk without SSDNC), and the result is shown in Figure 15. In an intuitive way, the results corresponding to $E=2$ are compared in Figure 16. In the same conditions, within the same mistuning levels (from 0 to $15 \%$ ), $\mathrm{AMF}_{1}$ and $\mathrm{AMF}_{3}$ vary, respectively, $78.50 \%$ (between 1 and 1.7850) and 41.39\% (between 1 and 1.4139). Then, we can conclude that owing to the SSDNC, the forced response of the blisk is less sensitive to mistuning, which means that the SSDNC can improve the robustness of the mistuned blisk.

5.4. Mistuning of the Capacitance Ratio. For a blisk with the SSDNC, there would be small discrepancies in the inherent capacitances of different piezo-patches or the negative capacitances in the circuit; hence, mistuning may occur on the capacitance ratios in different sectors. The mistuning level of the capacitance ratio $\chi_{\mathrm{c}}$ in sector $j$ is defined as $\delta \chi_{\mathrm{c}, j}$. It 
TABle 5: A mistuned sample of the capacitance ratio.

\begin{tabular}{lcccccccc}
\hline$j$ & 1 & 2 & 3 & 4 & 5 & 6 & 7 & 8 \\
$\delta \chi_{c, j}$ & -0.01198 & -0.00994 & -0.00349 & 0.00093 & -0.02548 & -0.00063 & -0.05489 & -0.02335 \\
\hline$j$ & 9 & 10 & 11 & 12 & 13 & 14 & 15 & 16 \\
$\delta \chi_{c, j}$ & -0.00985 & 0.00693 & 0.0695 & 0.03167 & 0.04206 & 0.01112 & -0.02066 & 0.01333 \\
\hline$j$ & 17 & 18 & 19 & 20 & 21 & 22 & 23 & -0.01302 \\
$\delta \chi_{c, j}$ & 0.03132 & 0.02821 & 0.00256 & -0.03639 & -0.03222 & -0.00915 & -0.01474 \\
\hline
\end{tabular}

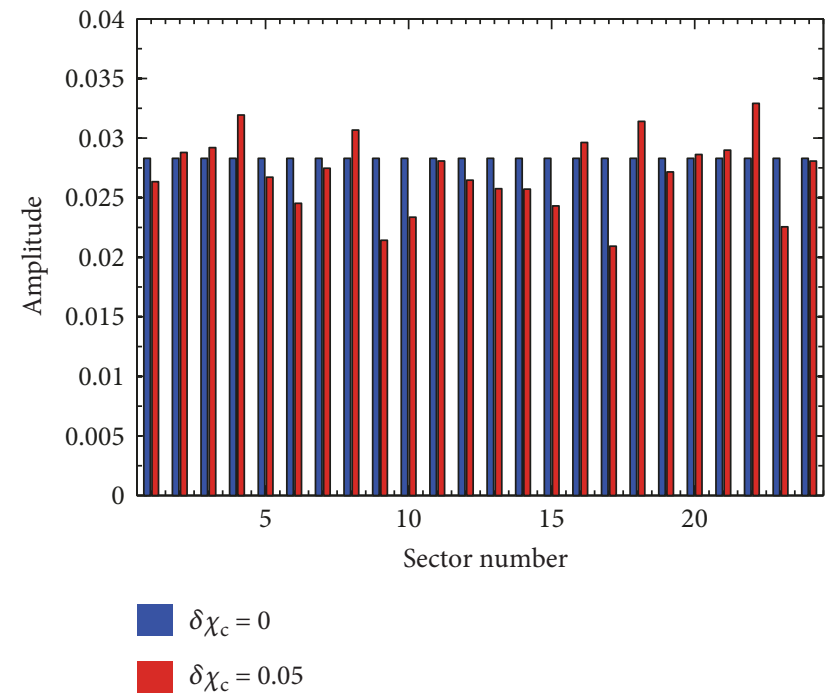

FIgURE 17: Response amplitude of different blades $(E=2)$.

should be noted that according to (3), when the capacitance is too close to 1, the nonlinear force induced by the SSDNC will approach infinity, and the system will even lose its stability. Therefore, in reality, the capacitance ratio is a compromise between vibration suppression and stability. In this mistuning analysis, the capacitance ratio is controlled to be no more than 0.96 . A random mistuning sample with capacitance ratio $\delta \chi_{c, j}$ is listed in Table 5. It follows a normal distribution with a mean value of 0 and its standard deviation is $5 \%$. The forced responses of the tuned blisk with the tuned and mistuned SSDNC are compared in Figure 17 when the system is excited by engine-order excitation $(E=2)$. It can be seen that the mistuned capacitance can also induce different forced responses from the blades.

The Monte Carlo simulation and statistical procedure are conducted using 500 samples in each condition, and the standard deviation of the mistuning level is changed from 0 to $5 \%$, with an increasing step of $0.25 \%$ (it should be noted that the blisk is tuned in the calculation). The estimations of $\mathrm{AMF}_{2}$ corresponding to different engine orders are given in Figure 18. As references, the AMFs corresponding to the tuned blisk with and without the SSDNC are also shown in Figure 18. It can be seen that, compared to the blisk with the tuned SSDNC, the forced response of the blisk with the mistuned SSDNC is amplified, but $\mathrm{AMF}_{2}$ is always much smaller than 1 . This result shows that even with the

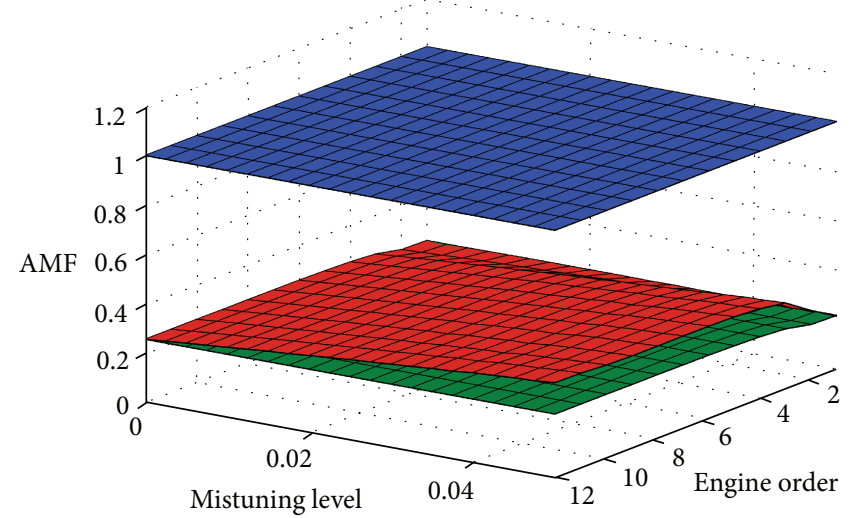

FIgURE 18: Estimations of AMFs corresponding to different conditions (blue: tuned blisk without SSDNC; green: tuned blisk with tuned SSDNC; red: tuned blisk with mistuned SSDNC).

mistuning of capacitance, the SSDNC can still reduce the vibration of the blisk effectively.

\section{Conclusions}

An NCMS method is proposed to predict the forced response of the blisk with the SSDNC. The method takes advantage of modal properties of the electromechanical system. Based on this method, a statistical analysis on the random mistuned blisk with the SSDNC was performed, and the proposed method overcame the main computational challenge in the analysis, arising from the nonlinearity and randomness of the system. The comparison with the MHBM-AFT method indicates that the NCMS method is precise enough and more efficient. The following conclusions were drawn.

The proposed NCMS method can accurately predict the forced response of the random mistuned blisk with the SSDNC, and the calculation cost can be considerably reduced. Although a lumped parameter model is used in the investigation, a high-fidelity model of the blisk can also be used in the analysis, and the efficiency of the NCMS method will be more prominent.

The statistical analysis results corresponding to different engine orders and mistuning levels show that the SSDNC can not only suppress the response amplification of the blades induced by random mistuning but also reduces the vibration localization of the blisk. In addition, it shows that the vibration-suppression performance of the SSDNC is 
insensitive to the mistuning of the mechanical parameters of the blisk.

The mistuning of the inherent capacitances of piezopatches and the negative capacitances in the circuit may reduce the effectiveness of vibration suppression by the SSDNC; however, the SSDNC can still reduce the vibration of the blisk considerably. The results will be of considerable significance in overcoming the problem of vibration magnification induced by the inevitable random mistuning of blisks in aeroengines.

In reality, the blade is usually much stiffer than the piezoelectric patch, so the geometrical parameters and distribution of the piezoelectric patches should be optimized to insure an acceptable electromechanical coupling factor and force factor. Therefore, an important future work is to study the empirical values for the coupling factor and force factor based on high fidelity finite element models.

\section{Data Availability}

The data used to support the findings of this study are available from the corresponding author upon request.

\section{Conflicts of Interest}

The authors declare that they have no conflicts of interest.

\section{Acknowledgments}

The authors would like to acknowledge the support of the National Natural Science Foundation of China (Grant nos. 51675022, 11702011), and Fan Yu would like to acknowledge the support of the China Postdoctoral Science Foundation (Grant no. 2018T110032 and 2017M610741).

\section{References}

[1] M. P. Castanier and C. Pierre, "Modeling and analysis of mistuned bladed disk vibration: current status and emerging directions," Journal of Propulsion and Power, vol. 22, no. 2, pp. 384-396, 2006.

[2] M. P. Castanier and C. Pierre, "Using intentional mistuning in the design of turbomachinery rotors," AIAA Journal, vol. 40, no. 10, pp. 2077-2086, 2002.

[3] C. M. Firrone and S. Zucca, Modelling Friction Contacts in Structural Dynamics and Its Application to Turbine Bladed Disks, INTECH, 2011.

[4] J. H. Griffin, "A review of friction damping of turbine blade vibration," International Journal of Turbo and Jet Engines, vol. 7, no. 3-4, pp. 297-307, 1990.

[5] A. Herzog, M. Krack, L. P.-v. Scheidt, and J. Wallaschek, "Comparison of two widely-used frequency-time domain contact models for the vibration simulation of shrouded turbine blades," in ASME Turbo Expo 2014: Turbine Technical Conference and Exposition, p. V07BT33A018, Düsseldorf, Germany, 2014, American Society of Mechanical Engineers.

[6] D. Laxalde, F. Thouverez, J.-J. Sinou, and J.-P. Lombard, "Qualitative analysis of forced response of blisks with friction ring dampers," European Journal of Mechanics - A/Solids, vol. 26, no. 4, pp. 676-687, 2007.
[7] E. P. Petrov and D. J. Ewins, "State-of-the-art dynamic analysis for non-linear gas turbine structures," Proceedings of the Institution of Mechanical Engineers, Part G: Journal of Aerospace Engineering, vol. 218, no. 3, pp. 199-211, 2004.

[8] N. Jalili, Piezoelectric-Based Vibration Control: From Macro to Micro/Nano Scale Systems, Springer Science \& Business Media, 2009.

[9] R. L. Forward, "Electronic damping of vibrations in optical structures," Applied Optics, vol. 18, no. 5, pp. 690-697, 1979.

[10] N. W. Hagood and A. Von Flotow, "Damping of structural vibrations with piezoelectric materials and passive electrical networks," Journal of Sound and Vibration, vol. 146, no. 2, pp. 243-268, 1991.

[11] J. Tang and K. W. Wang, "Vibration control of rotationally periodic structures using passive piezoelectric shunt networks and active compensation," Journal of Vibration and Acoustics, vol. 121, no. 3, pp. 379-390, 1999.

[12] J. Tang and K. W. Wang, "Active-passive hybrid piezoelectric networks for vibration control: comparisons and improvement," Smart Materials and Structures, vol. 10, no. 4, pp. 794-806, 2001.

[13] J. Liu, L. Li, P. Deng, and C. Li, "A comparative study on the dynamic characteristics of bladed disks with piezoelectric network and piezoelectric shunt circuit," in ASME Turbo Expo 2016: Turbomachinery Technical Conference and Exposition, p. V07AT32A012, Seoul, Republic of Korea, 2016.

[14] H. Yu and K. W. Wang, "Piezoelectric networks for vibration suppression of mistuned bladed disks," Journal of Vibration and Acoustics, vol. 129, no. 5, p. 559, 2007.

[15] H. Yu and K. W. Wang, "Vibration suppression of mistuned coupled-blade-disk systems using piezoelectric circuitry network," Journal of Vibration and Acoustics, vol. 131, no. 2, article 021008, 2009.

[16] B. Mokrani, R. Bastaits, M. Horodinca et al., "Parallel piezoelectric shunt damping of rotationally periodic structures," Advances in Materials Science and Engineering, vol. 2015, 12 pages, 2015.

[17] B. Zhou, F. Thouverez, and D. Lenoir, "Essentially nonlinear piezoelectric shunt circuits applied to mistuned bladed disks," Journal of Sound and Vibration, vol. 333, no. 9, pp. 2520-2542, 2014.

[18] C. Richard, D. Guyomar, D. Audigier, and H. Bassaler, "Enhanced semi-passive damping using continuous switching of a piezoelectric device on an inductor," in Smart Structures and Materials 2000: Damping and Isolation, pp. 288-299, Newport Beach, CA, USA, 2000.

[19] C. Richard, D. Guyomar, D. Audigier, and G. Ching, "Semipassive damping using continuous switching of a piezoelectric device," in Smart Structures and Materials 1999: Passive Damping and Isolation, pp. 104-111, Newport Beach, CA, USA, 1999.

[20] H. Ji, J. Qiu, L. Cheng, and H. Nie, "Semi-active vibration control based on unsymmetrical synchronized switch damping: analysis and experimental validation of control performance," Journal of Sound and Vibration, vol. 370, pp. 1-22, 2016.

[21] M. Neubauer, X. Han, and S. M. Schwarzendahl, "Enhanced switching law for synchronized switch damping on inductor with bimodal excitation," Journal of Sound and Vibration, vol. 330, no. 12, pp. 2707-2720, 2011.

[22] M. Neubauer, X. Han, and J. Wallaschek, "On the maximum damping performance of piezoelectric switching techniques," 
Journal of Intelligent Material Systems and Structures, vol. 24, no. 6, pp. 717-728, 2013.

[23] E. Lefeuvre, A. Badel, L. Petit, C. Richard, and D. Guyomar, "Semi-passive piezoelectric structural damping by synchronized switching on voltage sources," Journal of Intelligent Material Systems and Structures, vol. 17, no. 8-9, pp. 653660, 2006.

[24] K. Makihara, J. Onoda, and K. Minesugi, "A self-sensing method for switching vibration suppression with a piezoelectric actuator," Smart Materials and Structures, vol. 16, no. 2, pp. 455-461, 2007.

[25] H. Ji, J. Qiu, J. Cheng, and D. Inman, “Application of a negative capacitance circuit in synchronized switch damping techniques for vibration suppression," Journal of Vibration and Acoustics, vol. 133, no. 4, article 041015, 2011.

[26] A. Chérif, C. Richard, D. Guyomar, S. Belkhiat, and M. Meddad, "Simulation of multimodal vibration damping of a plate structure using a modal SSDI-max technique," Journal of Intelligent Material Systems and Structures, vol. 23, no. 6, pp. 675-689, 2012.

[27] D. Guyomar and A. Badel, "Nonlinear semi-passive multimodal vibration damping: an efficient probabilistic approach," Journal of Sound and Vibration, vol. 294, no. 1-2, pp. 249-268, 2006.

[28] H. Ji, J. Qiu, K. Zhu, and A. Badel, "Two-mode vibration control of a beam using nonlinear synchronized switching damping based on the maximization of converted energy," Journal of Sound and Vibration, vol. 329, no. 14, pp. 27512767, 2010.

[29] D. D’Assunção and C. De Marqui Junior, “Applied selfpowered semi-passive control for a 2-degree-of-freedom aeroelastic typical section using shunted piezoelectric materials," Journal of Intelligent Material Systems and Structures, vol. 26, no. 4, pp. 373-385, 2015.

[30] M. Lallart, É. Lefeuvre, C. Richard, and D. Guyomar, "Self-powered circuit for broadband, multimodal piezoelectric vibration control," Sensors and Actuators A: Physical, vol. 143, no. 2, pp. 377-382, 2008.

[31] E. M. Qureshi, X. Shen, and L. Chang, "Self-powered synchronized switch damping on negative capacitance for broadband vibration suppression of flexible structures," International Journal of Applied Electromagnetics and Mechanics, vol. 49, no. 1, pp. 105-121, 2015.

[32] J. L. Kauffman and G. A. Lesieutre, "Piezoelectric-based vibration reduction of turbomachinery bladed disks via resonance frequency detuning," AIAA Journal, vol. 50, no. 5, pp. 11371144, 2012.

[33] G. Lopp and J. L. Kauffman, "Switch triggers for optimal vibration reduction via resonance frequency detuning," in ASME Turbo Expo 2014: Turbine Technical Conference and Exposition, p. V07AT34A007, Düsseldorf, Germany, 2014.

[34] J. Liu, L. Li, X. Huang, and L. Jezequel, "Dynamic characteristics of the blisk with synchronized switch damping based on negative capacitor," Mechanical Systems and Signal Processing, vol. 95, no. 1, pp. 425-445, 2017.

[35] D. Laxalde, L. Salles, L. Blanc, and F. Thouverez, "Non-linear modal analysis for bladed disks with friction contact interfaces," in ASME Turbo Expo 2008: Power for Land, Sea, and Air, pp. 457-467, Berlin, Germany, 2008.

[36] D. Laxalde and F. Thouverez, "Complex non-linear modal analysis for mechanical systems: application to turbomachinery bladings with friction interfaces," Journal of Sound and Vibration, vol. 322, no. 4-5, pp. 1009-1025, 2009.

[37] C. Joannin, B. Chouvion, F. Thouverez, J. P. Ousty, and M. Mbaye, "A nonlinear component mode synthesis method for the computation of steady-state vibrations in nonconservative systems," Mechanical Systems and Signal Processing, vol. 83, pp. 75-92, 2017.

[38] P. Wang and L. Li, "Parametric dynamics of mistuned bladed disk," in ASME Turbo Expo 2014: Turbine Technical Conference and Exposition, Düsseldorf, Germany, 2014.

[39] M. Krack, L. Panning-von Scheidt, and J. Wallaschek, "A method for nonlinear modal analysis and synthesis: application to harmonically forced and self-excited mechanical systems," Journal of Sound and Vibration, vol. 332, no. 25, pp. 6798-6814, 2013.

[40] C. Joannin, B. Chouvion, and F. Thouverez, "A reduced-order modeling technique for the study of non-linear vibrations in dissipative systems," in ISMA2016 - International Conference on Noise and Vibration Engineering, pp. 2671-2681, Leuven, Belgium, 2016.

[41] P.-c. Deng, L. Li, and C. Li, "Study on vibration of mistuned bladed disk with bi-periodic piezoelectric network," Proceedings of the Institution of Mechanical Engineers, Part G: Journal of Aerospace Engineering, vol. 231, no. 2, pp. 350-363, 2016.

[42] L. Li, P. Deng, J. Liu, and C. Li, "Theoretical study of the vibration suppression on a mistuned bladed disk using a bi-periodic piezoelectric network," International Journal of Turbo \& JetEngines, vol. 35, no. 1, pp. 17-28, 2018. 


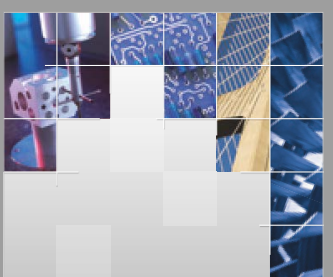

\section{Enfincering}
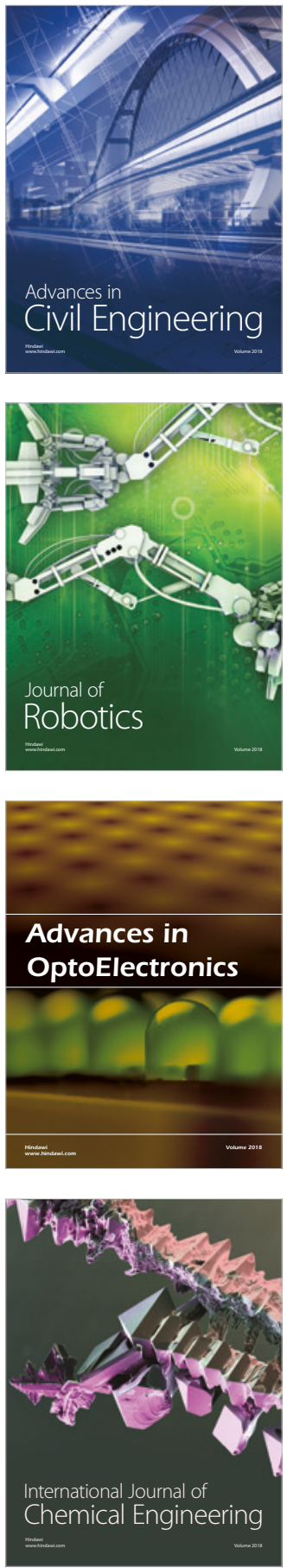

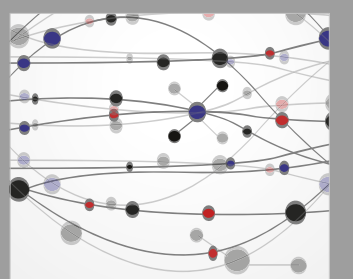

\section{Rotating \\ Machinery}

The Scientific World Journal

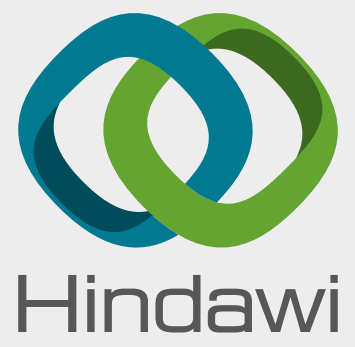

Submit your manuscripts at

www.hindawi.com
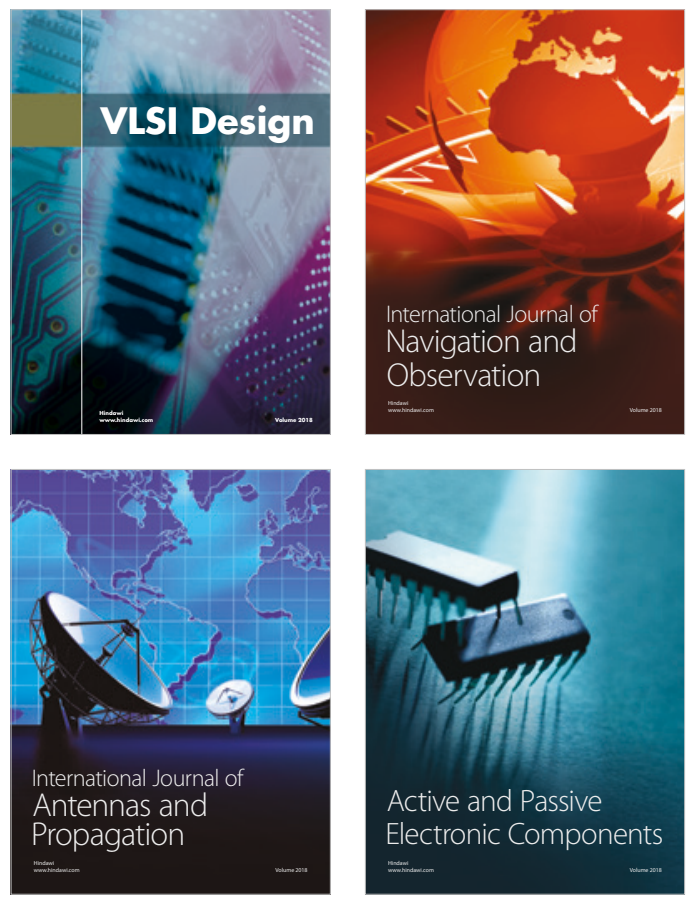
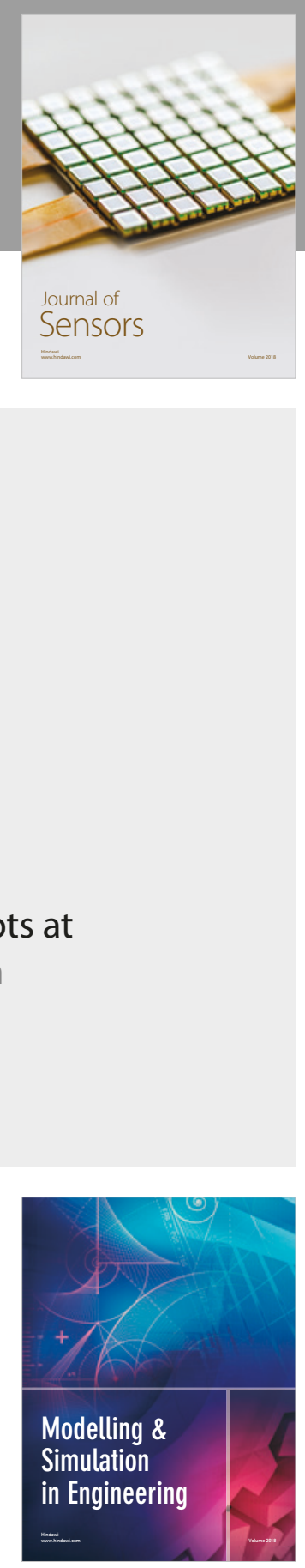

\section{Advances \\ Multimedia}
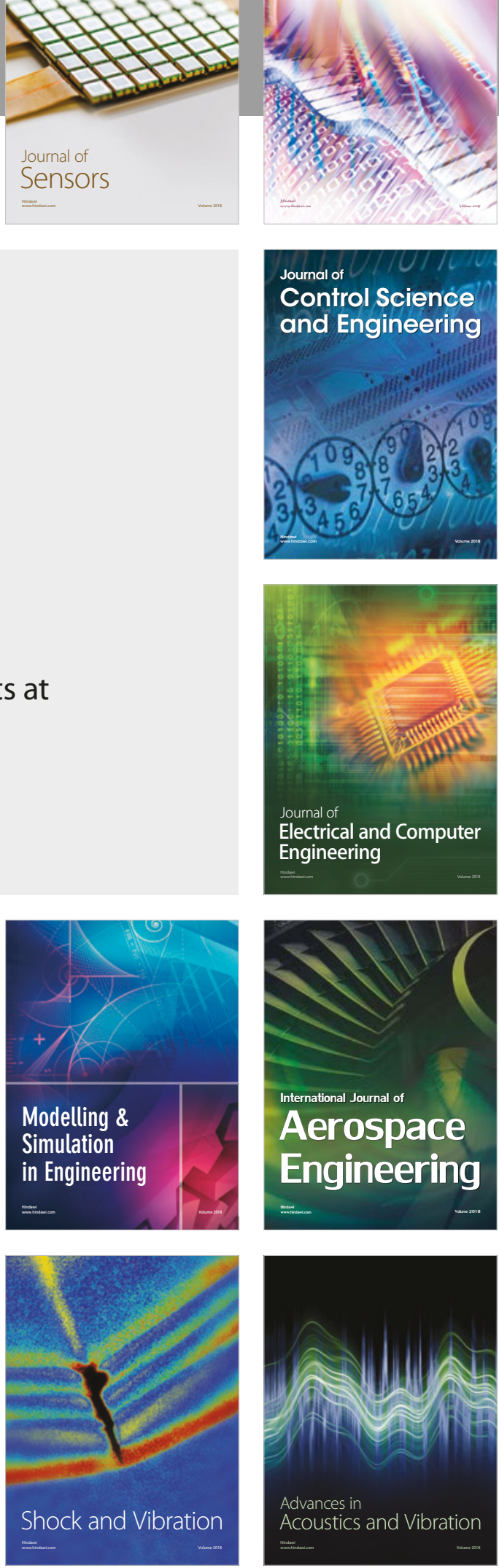